\title{
Testing asteroseismology with Gaia DR2: Hierarchical models of the Red Clump
}

Oliver J. Hall ${ }^{1,2 \star}$, Guy R. Davies ${ }^{1,2}$, Yvonne P. Elsworth ${ }^{1,2}$, Andrea Miglio ${ }^{1,2}$, Timothy R. Bedding ${ }^{3,2}$, Anthony G. A. Brown ${ }^{4}$, Saniya Khan ${ }^{1,2}$, Keith Hawkins ${ }^{5}$, Rafael A. García 6,7 , William J. Chaplin ${ }^{1,2}$, Thomas S. H. North ${ }^{1}$

${ }^{1}$ School of Physics and Astronomy, University of Birmingham, Edgbaston, Birmingham, B15 2TT, UK

${ }^{2}$ Stellar Astrophysics Centre, Department of Physics and Astronomy, Aarhus University, Ny Munkegade 120, 8000 Aarhus C, Denmark

${ }^{3}$ Sydney Institute for Astronomy, School of Physics, University of Sydney 2006, Australia

${ }^{4}$ Leiden Observatory, Leiden University, Niels Bohrweg 2, 2333 CA, Leiden, The Netherlands

${ }^{5}$ Department of Astrnomy, The University of Texas at Austin, 2515 Speedway Boulevard, Austin, TX 78712, USA

${ }^{6}$ IRFU, CEA, Université Paris-Saclay, F-91191 Gif-sur-Yvette, France

${ }^{7}$ AIM, CEA, CNRS, Université Paris-Saclay, Université Paris Diderot, Sorbonne Paris Cité, F-91191 Gif-sur-Yvette, France

Accepted XXX. Received YYY; in original form ZZZ

\begin{abstract}
Asteroseismology provides fundamental stellar parameters independent of distance, but subject to systematics under calibration. Gaia DR2 has provided parallaxes for a billion stars, which are offset by a parallax zero-point $\left(\varpi_{\mathrm{zp}}\right)$. Red Clump (RC) stars have a narrow spread in luminosity, thus functioning as standard candles to calibrate these systematics. This work measures how the magnitude and spread of the RC in the Kepler field are affected by changes to temperature and scaling relations for seismology, and changes to the parallax zero-point for Gaia. We use a sample of 5576 RC stars classified through asteroseismology. We apply hierarchical Bayesian latent variable models, finding the population level properties of the $\mathrm{RC}$ with seismology, and use those as priors on Gaia parallaxes to find $\varpi_{\text {zp }}$. We then find the position of the RC using published values for $\varpi_{\mathrm{zp}}$. We find a seismic temperature insensitive spread of the RC of $\sim 0.03$ mag in the 2MASS $K$ band and a larger and slightly temperature-dependent spread of $\sim 0.13 \mathrm{mag}$ in the Gaia $G$ band. This intrinsic dispersion in the $K$ band provides a distance precision of $\sim 1 \%$ for RC stars. Using Gaia data alone, we find a mean zero-point of $-41 \pm 10 \mu$ as. This offset yields RC absolute magnitudes of $-1.634 \pm 0.018$ in $K$ and $0.546 \pm 0.016$ in $G$. Obtaining these same values through seismology would require a global temperature shift of $\sim-70 \mathrm{~K}$, which is compatible with known systematics in spectroscopy.
\end{abstract}

Key words: parallax - asteroseismology - stars: fundamental parameters - stars: statistics

\section{INTRODUCTION}

Since the launch of CoRoT (Baglin et al. 2006) and Kepler (Borucki et al. 2010), the use of asteroseismology - the study of stars' internal physics by observing their modes of oscillation - has become a crucial tool for testing fundamental stellar properties. The large quantity of long timeseries photometry from these missions (Chaplin \& Miglio 2013), and its distance independent nature, have allowed for mea-

^ E-mail: ojh251@bham.ac.uk (OJH) sures of precise stellar radii and masses for both red giant stars (Hekker et al. 2011; Huber et al. 2011, 2014; Mathur et al. 2016; Pinsonneault et al. 2014; Pinsonneault et al. 2018; Yu et al. 2018) and main sequence stars (Chaplin et al. 2010, 2011, 2014), studies of exoplanets and exoplanet hosts (Christensen-Dalsgaard et al. 2010; Batalha et al. 2011; Huber et al. 2013a,b; Chaplin et al. 2013; Silva Aguirre et al. 2015), internal \& external stellar rotation (Beck et al. 2012; Deheuvels et al. 2012, 2014; Mosser et al. 2012b; Davies et al. 2015), ages of stellar populations (Miglio et al. 2009, 2013; Casagrande et al. 2014, 2016; Stello et al. 2015), and classi- 
fications of stellar types (Bedding et al. 2011; Mosser et al. 2012a, 2015; Stello et al. 2013; Vrard et al. 2016; Elsworth et al. 2017), among others.

Many of these works rely on the so-called 'direct method': the use of seismic scaling relations related to the two fundamental oscillation parameters, $v_{\max }$, the frequency of maximum power of the oscillation mode envelope, and $\Delta v$, the spacing between two oscillation modes of equal radial degree. These properties are individually proportional to mass, radius and temperature, and when combined and scaled with solar values, can provide measures of stellar mass, radius and surface gravity (Kjeldsen \& Bedding 1995). As such, stellar properties obtained through seismology depend on temperature as well as on the seismic parameters. Besides the direct method, results from seismology can also be obtained by comparing global seismic properties with a grid of models, referred to as 'grid modelling', and can be expanded to 'detailed modelling', which directly fits observed seismic mode frequencies to the grids (Metcalfe et al. 2012, 2014; Silva Aguirre et al. 2013, 2015; Davies et al. 2016; Lund et al. 2017).

The seismic scaling relations have been thoroughly tested through interferometry (White et al. 2013), astrometry (Huber et al. 2017), eclipsing binaries (Gaulme et al. 2016), and open clusters (Miglio et al. 2012). Theoretically motivated corrections to the $\Delta v$ and $v_{\max }$ scaling relations have been proposed to depend on $T_{\text {eff }}$, metallicity, and evolutionary state (Miglio et al. 2012; Sharma et al. 2016), and it is known that a small correction for the mean molecular weight could be needed for the $v_{\max }$ scaling relation (Belkacem et al. 2013; Viani et al. 2017).

When using the direct method, effective temperatures from spectroscopic analysis are often used (e.g. the APOKASC catalogue; Pinsonneault et al. (2014); Pinsonneault et al. (2018)). However depending on the atmospheric models and temperature scales applied in spectroscopic analysis, inferred values for $T_{\text {eff }}$ can vary up to $\sim 170 \mathrm{~K}$ for Core Helium-Burning (CHeB) stars (Slumstrup et al. 2019). While Bellinger et al. (2019) have recently shown that these systematic uncertainties can be mitigated through the use of grid modelling for main-sequence and sub-giant stars, the question of which temperature scale for spectroscopy obtains the best value for $T_{\text {eff }}$ remains open.

Seismic observations can be combined with distance dependent observations, such as astrometry, to improve and calibrate results. The second data release (DR2) of the astrometric Gaia mission (Gaia Collaboration et al. 2018) recently has provided data for a sample of over one billion targets, with uncertainties largely improved from the first data release (DR1, TGAS Gaia Collaboration et al. 2016), allowing for a broader range of science and calibrations (Zinn et al. 2018). With DR2 Lindegren et al. (2018) suggested a mean global parallax zero-point offset of $-29 \mu$ as, in the sense that Gaia parallaxes are too small, using a quasar sample, although it should be noted that the offset varies as a function of colours, magnitude and position on the sky. Arenou et al. (2018) computed the parallax difference between DR2 and existing catalogues, as well as prior data for individual targets, and found these on average to be the same order of magnitude as the Lindegren et al. (2018) zero-point. Riess et al. (2018) used Cepheid variables to derive a zero-point offset of $-46 \pm 13 \mu \mathrm{as}$, Stassun \& Torres (2018) used Eclipsing Binaries to find a zero-point of $-83 \pm 33 \mu$ as, and Zinn et al. (2018) compared parallaxes to seismic radii to identify a colour- and magnitude-dependent offset of $-52.8 \pm 2.4$ (stat. $) \pm 1$ (syst.) $\mu$ as for red giant branch stars in the Kepler field. Finally, using analysis of individual seismic mode frequencies for 93 dwarf stars, Sahlholdt \& Silva Aguirre (2018) reported an offset in estimated stellar radii equal to a parallax offset of $-35 \pm 16 \mu$ as. As the parallax zero-point offset is known to vary with magnitude, colour, and position in the sky, the differences between these values for the zero-point are expected. Understanding how we quantify the offset is crucial if we want to use Gaia to calibrate asteroseismology and other methods.

One method of testing independent sets of measurements is calculating an observable astronomical property. An example of such a property is the luminosity of the 'Red Clump' (RC), an overdensity of red giant stars on the HRdiagram, in bands of absolute magnitude. When stars of masses around $0.7 \lesssim M / M_{\odot} \lesssim 1.9$ (for $[\mathrm{M} / \mathrm{H}] \simeq 0.07$, upper limit subject to change with metallicity) ignite helium in their cores, they undergo the He-flash. The He-burning core masses are very similar for these stars, and as their luminosity is mainly determined by the core mass, they will all have similar luminosities, creating a clump of stars on the HR-diagram (Girardi 2016, and references therein). Further differences in luminosity and temperature are then effects of metallicity and envelope mass, and thus the Clump has a relatively small spread. Stars at lower masses and low metallicities form a horizontal branch at a luminosity similar to the RC, whereas stars of masses just above the limit for the He-flash lie at a slightly lower luminosity, forming a Secondary Red Clump (2CL, Girardi 1999). At even higher masses, the luminosity becomes a function of stellar mass, and these stars form a vertical structure in the HR-diagram during their $\mathrm{CHeB}$ phase.

The luminosity of the RC overdensity may be used as a standard candle given constraints on mass and metallicity (Cannon 1970), and has recently been used to calibrate Gaia DR1 parallaxes (Davies et al. 2017). Also using Gaia DR1 parallaxes, Hawkins et al. (2017) (hereafter H17) found precise measurements for the RC luminosity in various passbands, including the 2MASS $K$ band, which minimised the spread in luminosity due to mass and metallicity (Salaris \& Girardi 2002). With Gaia DR2's improved parallax uncertainties and reduced systematic offset, now is a good time to revisit the $\mathrm{RC}$ as a calibrator.

In this work we investigate systematics in both asteroseismology and Gaia simultaneously, to see how differences in assumptions for one influence inferences of the other. Using a sample of over 5500 Kepler Red Giant stars in the $\mathrm{RC}$ for which parallaxes and seismology are available, we measure the position of the RC population in absolute magnitude in the 2MASS (Skrutskie et al. 2006) $K$ band, and the Gaia $G$ band. We do this using seismology and parallax (with photometry) independently. Since the distribution of $\mathrm{RC}$ stars should be the same for this population, independent of method, a (dis)agreement of the measured positions and spreads of the RC using two independent methods sheds light on systematics in both. For the seismic method, we test the influence of the temperature scale used to obtain 
the values of $T_{\text {eff }}$ fed into seismic scaling relations, as well as the impact of corrections to the $\Delta v$ scaling relation. For the Gaia method, we study how changes in the parallax zeropoint offset for Gaia DR2 impact the inferred luminosity of the $\mathrm{RC}$

This paper is laid out as follows: Section 2 discusses how the data were obtained, and the theory used to calculate our observables. Section 3 discusses how we use hierarchical Bayesian modelling to study the RC. We present our results in Section 4 and discuss them in context of similar work in Section 5, and present our conclusions in Section 6.

\section{DATA}

Our aim is to find the intrinsic position and spread of the Red Clump in absolute magnitude for various passbands using two approaches: one using a distance-independent luminosity calculated from asteroseismology, and the other using a magnitude inferred from photometry and Gaia DR2 parallaxes. Since the number of stars with asteroseismic data is significantly lower than those with data in Gaia DR2, this limits our sample.

For our asteroseismic sample, we used the catalogue of 16,094 oscillating Kepler red giants by Yu et al. (2018) (hereafter Y18), which contains global oscillation parameters $v_{\max }$ and $\Delta v$, as well as broad evolutionary state classifications, effective temperatures $T_{\text {eff }}$ and metallicities $[\mathrm{Fe} / \mathrm{H}]$ taken from Mathur et al. (2017).

We re-considered the classification of all stars labelled as $\mathrm{CHeB}$ in the $\mathrm{Y} 18$ catalogue using the method presented in Elsworth et al. (2017). This uses the structure of dipolemode oscillations in the power spectra to classify stars as belonging to the 2CL, the Red Giant Branch (RGB), or the RC. We obtained light curves for 7437 stars labelled as $\mathrm{CHeB}$ in Y18, from two sources: the so-called KASOC light curves (Handberg \& Lund 2014) ${ }^{1}$ and the KEPSEISMIC light curves (García et al. 2011) ${ }^{2}$. The latter have been produced with larger photometric masks to ensure a better stability at low frequencies, and have been gap-filled using in-painting techniques (García et al. 2014; Jofré et al. 2015).

Of these 7437 stars, we found that 5668 are RC, 737 are 2CL, and no classification could be found for 499 stars. Notably, 533 stars were found to be RGB, disagreeing with the classification listed in Y18. This should be discussed in future work, but for the sake of internal consistency of our classifications we have chosen to adopt Elsworth et al. (2017) classification in this work.

It should be noted that our classification does not specifically account for low-mass, low-metallicity horizontal branch stars, which are therefore expected to be retained in our sample, but are not expected to significantly affect the result as they have similar luminosities to the $\mathrm{RC}$, and no extensive horizontal structure is present on the HR diagram of the Y18 catalogue, or our subsample thereof (see Figure 1). A fraction of the newly classified stars had masses reported in Y18 as much higher than we would expect for a

\footnotetext{
1 Freely distributed at the KASOC webpage (http://kasoc. phys.au.dk)

2 Freely distributed at the MAST website (https://archive. stsci.edu/prepds/kepseismic/)
}

RC star. In order to exclude these from our sample, we apply a liberal cut for clump-corrected seismic masses of over $2.2 M_{\odot}$, excluding 92 stars from our sample.

To obtain our astrometric sample, we cross-matched the RC stars we selected from the Y18 sample with the Gaia

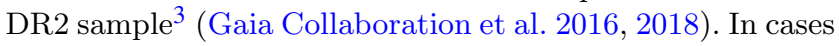
of duplicate sources for a given KIC, we selected the star with the lowest angular separation to the target. We did not apply any truncation of the sample based on parallax uncertainty or negative parallax, since this is known to introduce a parallax dependent bias (Luri et al. 2018).

The parallaxes $(\hat{\varpi})$ and parallax uncertainties $\left(\sigma_{\hat{\varpi}}\right)$ make up our astrometric set of observables. We obtained the apparent magnitudes $(\hat{m})$ and their uncertainties $\left(\sigma_{\hat{m}}\right)$ from the 2MASS survey for the $K$ band (Skrutskie et al. 2006) and Gaia DR2 for the Gaia $G$ band, and removed stars that do not have photometry or uncertainties on magnitude in 2MASS. Comparing the magnitude zero-points for the Gaia $G, G_{\mathrm{BP}}$ and $G_{\mathrm{RP}}$ bands, Casagrande \& VandenBerg (2018b) found indication of a magnitude-dependent zeropoint offset in the Gaia $G$ band magnitudes in the range of 6 mag $\lesssim \mathrm{G} \lesssim 16.5 \mathrm{mag}$, corrected as

$G^{\mathrm{corr}}=0.0505+0.9966 G$,

where $G$ is our uncorrected Gaia $G$ band magnitude. This correction is small, and corresponds to 30 mmag over 10 magnitudes. We gave all our $G$ band magnitudes a generous uncertainty of $10 \mathrm{mmag}$, the typical uncertainty quoted in Gaia Collaboration et al. (2018) for $G=20$, in order to account for any additional uncertainty incurred by the above correction. It should be noted that a similar relation for the correction of $G$ band magnitudes is presented in Maíz Apellániz \& Weiler (2018). This correction places magnitudes about 30 mmag higher than when using the Casagrande \& VandenBerg (2018b) correction in the applicable magnitude range. We expect the scale of this systematic offset to have a negligible impact on our results, and therefore adopt the Casagrande \& VandenBerg (2018b) correction in this work for consistency with our chosen $G$ band extinction coefficients and bolometric corrections.

Our model also uses an extinction for each star in each band. Reddening values are taken from the Green et al. (2018) three-dimensional dustmap under the assumption that the distance to the object is that given by Bailer-Jones et al. (2018). We note that this is not expected to bias our results towards a previous measure of distance, because the spread in the obtained reddening values, regardless of choice of distance value, falls well within the spread of the prior set on these values in our model. We converted reddening to the band-specific extinction $\hat{A}_{\lambda}$ using extinction coefficients unique to the Green et al. (2018) map for the $K$ band ${ }^{4}$. For the Gaia $G$-band we calculated our band-specific extinction using the mean extinction coefficient presented in Casagrande \& VandenBerg (2018b), after converting our reddening value to a measure of $E(B-V)$ following the conventions presented in Green et al. (2018).

\footnotetext{
3 We make use of the of the https://www.gaia-kepler.fun crossmatch database created by Megan Bedell for this purpose.

4 These coefficients can be found with the Green et al. (2018) usage notes.
} 


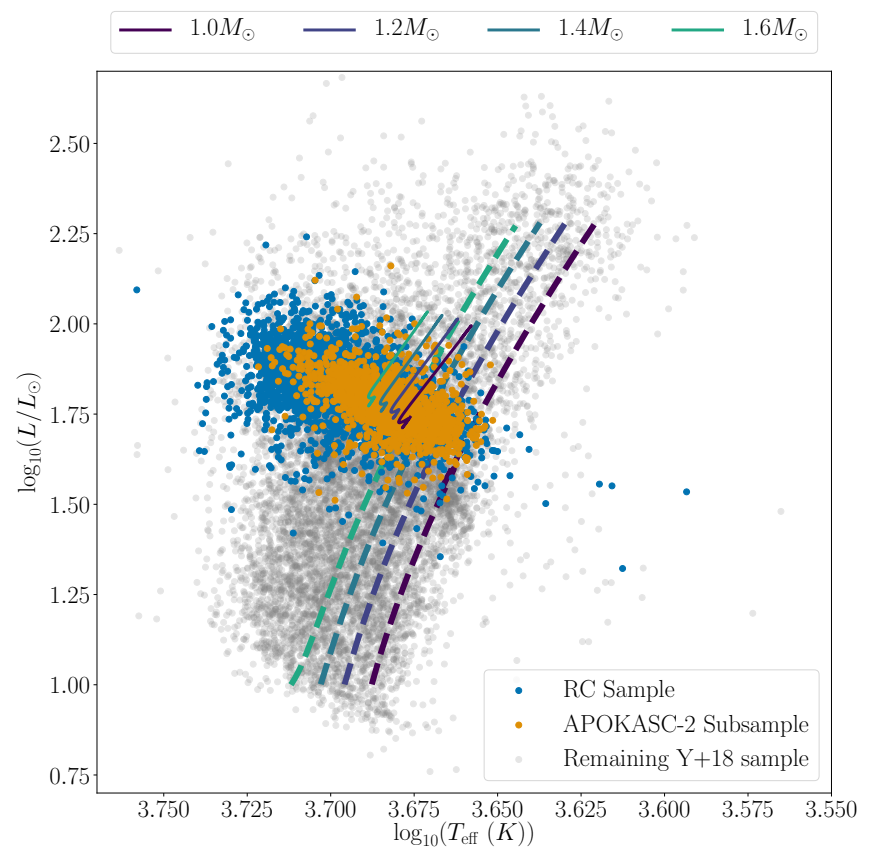

Figure 1. HR diagram illustrating the data in our final set of 5576 stars overlaid on the Y18 sample, along with evolutionary tracks from MESA (Paxton et al. 2011, 2013, 2015)(for details about the physical inputs of the models see Khan et al. 2018). The stars in the Y18 sample not in our final selection are in grey. Plotted on top in blue are the stars that in our final sample where the subsample of stars with temperatures reported in APOKASC2 (Pinsonneault et al. 2018) are shown in orange. Evolutionary tracks are plotted for for masses ranging between 1.0 and 1.6 solar masses for a metallicity of $Z=0.01108$ and helium content of $Y=0.25971$. The dashed lines indicate the Red Giant Branch, whereas the solid lines indicate the main Core Helium Burning stage of the tracks (the Helium flash (and subflashes) are not included).

The final sample contains $5576 \mathrm{RC}$ stars, with minimal contamination from the $2 \mathrm{CL}$ or the $\mathrm{RGB}$, and covers a magnitude range of $\sim 8$ to $\sim 16 \mathrm{mag}$ in $G$ and $\sim 6$ to $\sim 14$ mag in $K$. Note that for this magnitude range we expect the Gaia DR2 catalogue to be practically complete, and do not need to apply any selection functions in magnitude. The data are shown in Figure 1 in an HR diagram overlaid on the full Y18 sample.

\subsection{The APOKASC-2 subsample}

We used temperatures from Mathur et al. (2017), a catalogue compiling temperatures from a diverse set of papers including work with spectroscopy, photometry, and some asteroseismology. In order to investigate the impact of using differing temperature sources on our results, we also included runs on a subsample of 1637 stars that had $T_{\text {eff }}$ values reported in the APOKASC-2 catalogue (Pinsonneault et al. 2014; Pinsonneault et al. 2018). When calculating seismic properties from these data, we only changed the values for $T_{\text {eff }}$ to our new APOKASC-2 values. In Figure 2 we compare the distributions in $T_{\text {eff }}$, mass, radius and $[\mathrm{Fe} / \mathrm{H}]$ of the $\mathrm{Y} 18$ $\mathrm{RC}$ sample and the APOKASC-2 subsample. Also shown is the distribution of the APOKASC-2 temperatures, which are overall lower than the Y18 temperatures, and the distributions in mass and radius calculated through the direct method for these temperatures. Overall the APOKASC-2 subsample represents a lower temperature population, with its most distinct difference being in $T_{\text {eff }}$ and $[\mathrm{Fe} / \mathrm{H}]$.

\subsection{Obtaining the seismic sample}

The two global observable seismic parameters, $v_{\max }$ and $\Delta v$, scale with fundamental stellar properties as (Brown et al. 1991; Kjeldsen \& Bedding 1995):

$\frac{v_{\max }}{v_{\max \odot}} \simeq\left(\frac{M}{M_{\odot}}\right)\left(\frac{R}{R_{\odot}}\right)^{-2}\left(\frac{T_{\mathrm{eff}}}{T_{\mathrm{eff} \odot}}\right)^{-1 / 2}$ and

$\frac{\Delta v}{\Delta v_{\odot}} \simeq\left(\frac{M}{M_{\odot}}\right)^{1 / 2}\left(\frac{R}{R_{\odot}}\right)^{-3 / 2}$

where $M$ is the stellar mass, $R$ is the radius, $T_{\text {eff }}$ is the effective temperature, and $\odot$ indicates a solar value. In this work we used $v_{\max \odot}=3090 \pm 30 \mu \mathrm{Hz}, \Delta v_{\odot}=135.1 \pm 0.1 \mu \mathrm{Hz}$ and $T_{\text {eff } \odot}=5777 \mathrm{~K}$ (Huber et al. 2011). By rearranging these scaling relations, we can obtain stellar surface gravity and radius as

$\frac{g}{g_{\odot}} \simeq \frac{v_{\max }}{v_{\max \odot}}\left(\frac{T_{\mathrm{eff}}}{T_{\mathrm{eff} \odot}}\right)^{1 / 2}$ and

$\frac{R}{R_{\odot}} \simeq\left(\frac{v_{\max }}{v_{\max \odot}}\right)\left(\frac{\Delta v}{f_{\Delta v} \Delta v_{\odot}}\right)^{-2}\left(\frac{T_{\mathrm{eff}}}{T_{\mathrm{eff} \odot}}\right)^{1 / 2}$

where the new term $f_{\Delta v}$ is a correction to the $\Delta v$ scaling relation in the notation of Sharma et al. (2016). We calculated $f_{\Delta v}$ as a function of $[\mathrm{Fe} / \mathrm{H}], T_{\mathrm{eff}}, v_{\max }, \Delta v$ and evolutionary state using interpolation in a grid of models (Sharma \& Stello 2016). For each perturbation of $T_{\text {eff }}$ we recalculated $f_{\Delta v}$, changing no other parameters. We only extracted the correction values $f_{\Delta v}$ from the models, and used the seismic parameters and temperature values from our original set, and not the results for these values returned from the grids, in the rest of this work. We did not include corrections for the $v_{\max }$ scaling relation, because these are more difficult to obtain theoretically (Belkacem et al. 2011), and are probably negligible (Brogaard et al. 2018). Note that Brogaard et al. (2018) found that using corrections by Rodrigues et al. (2017) delivers on average slightly smaller stellar properties than using Sharma \& Stello (2016) due to differences in how these methods treat the solar surface effect. Since we used a wide range of bolometric corrections for various temperature perturbations, the method by Rodrigues et al. (2017) would be too computationally expensive, and we thus elected to use Sharma \& Stello (2016), which may lead to differences of the order of $\sim 2 \%$ in radius than if we had used Rodrigues et al. (2017) (White et al. 2011). We discuss the impact of this on our work in Section 5.

In order to obtain absolute magnitudes for our sample, we used $T_{\text {eff }}$ and seismic radii, calculated using $\Delta v$ and $v_{\max }$ 

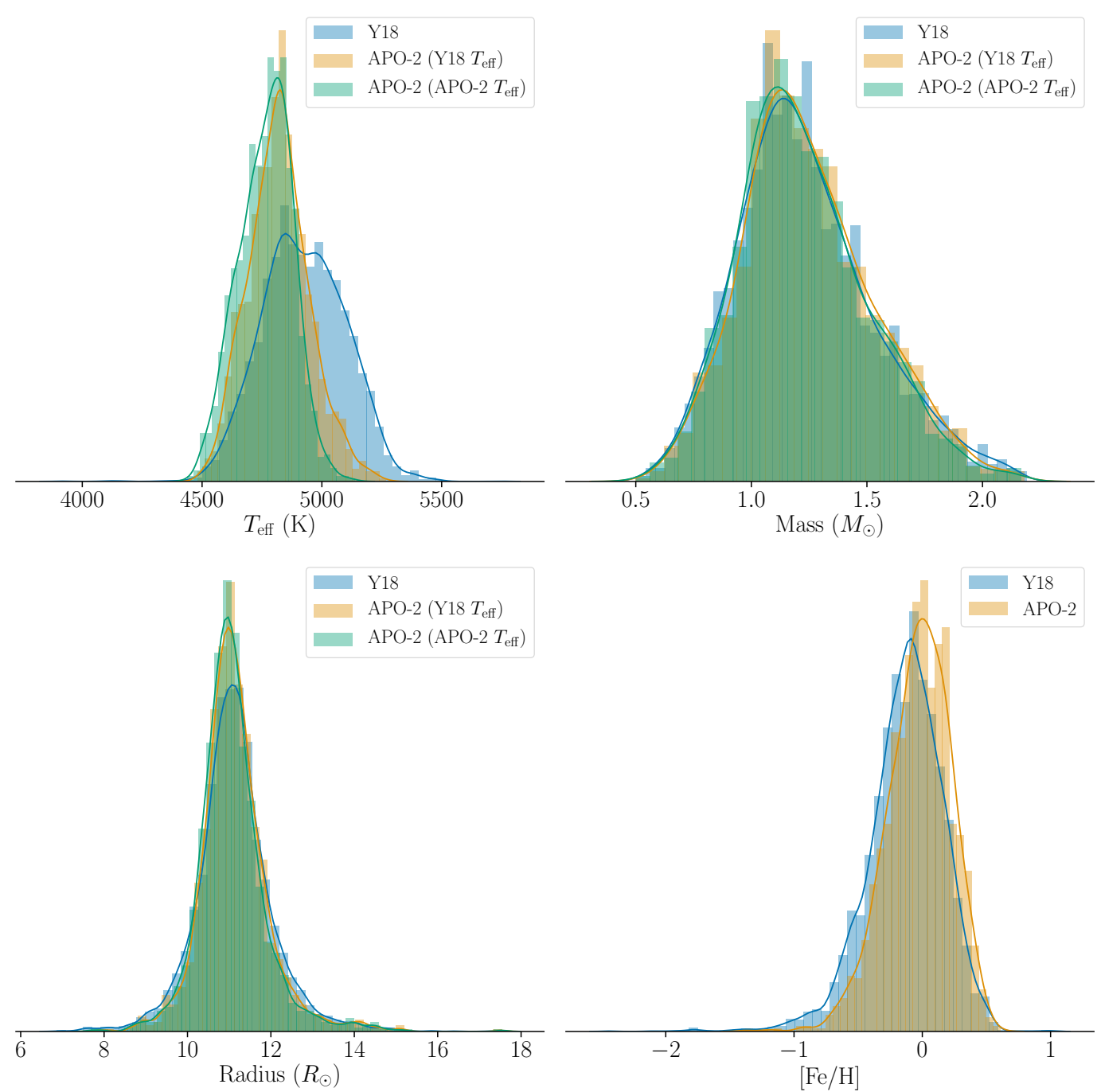

Figure 2. Distributions in $T_{\text {eff }}$, mass, radius and [Fe/H] of the RC sample (Yu et al. 2018) and the APOKASC-2 subsample (Pinsonneault et al. 2014; Pinsonneault et al. 2018). In green are the distribution of the APOKASC-2 temperatures, which are overall lower, and the distributions in mass and radius calculated through the direct method for these temperatures. In the labels, 'APO-2' represents a shorthand for APOKASC-2.

from the Y18 catalogue through equation (5), to calculate the stellar luminosity as

$L_{*}=4 \pi \sigma_{\mathrm{sb}} R^{2} T_{\mathrm{eff}}^{4}$.

Here $L_{*}$ is the luminosity of the star and $\sigma_{\mathrm{sb}}$ is the StefanBoltzmann constant. This was converted to a bolometric magnitude as in Casagrande \& VandenBerg (2014):

$M_{\mathrm{bol}}=-2.5 \log _{10}\left(L_{*} / L_{\odot}\right)+M_{\mathrm{bol} \odot}$,

where $L_{\odot}$ is the solar luminosity, and we have adopted $M_{\text {bol } \odot}=4.75$ (Casagrande \& VandenBerg 2014, 2018a,b). We calculated the bolometric correction (BC) in the 2MASS $K$ and Gaia $G$ bands with the method described by Casagrande \& VandenBerg $(2014,2018 \mathrm{a}, \mathrm{b})$ using $T_{\text {eff }},[\mathrm{Fe} / \mathrm{H}]$ and $\log g$, and without accounting for extinction. Since we are using a distance-independent measure of luminosity to calculate an absolute magnitude, accounting for this in the $\mathrm{BC}$ would bias our results. Because our method requires tweaking our values for $T_{\text {eff }}$, we recalculated the $\log g$ used to find the BC through the scaling relation in equation (4), as well as our values for $f_{\Delta v}$, for each different set of temperatures, and thus obtained a full set of bolometric corrections and corrections to the scaling relations for each temperature perturbation. Our values of absolute magnitude were then given by

$\hat{M}_{\lambda}=M_{\mathrm{bol}}-B C_{\lambda}$,

where $\lambda$ is the relevant band, $M_{\mathrm{bol}}$ is the bolometric luminosity and $B C_{\lambda}$ is the bolometric correction in that band. Uncertainties on $\hat{M}_{\lambda}$ were propagated through from the uncertainties on seismic parameters and effective temperatures, including those on the solar seismic parameters. Uncertainties on the BCs were estimated using a Monte Carlo method with 5000 iterations for 1000 randomly selected stars from our sample. We found an uncertainty of $0.3 \mathrm{mag}$ for all BCs 
in the $G$ band. For the $K$ band we found 0.05 mag for stars with a fractional temperature uncertainty of $<2.5 \%$, and 0.09 mag for those with larger fractional uncertainties on temperature. We discuss the systematic uncertainties on $f_{\Delta v}$ in Section 5.

\section{LOCATING THE RED CLUMP USING HIERARCHICAL BAYESIAN MODELLING}

In order to test systematics in asteroseismology and Gaia using the Red Clump (RC), we aim to find the location and spread of the RC in absolute magnitude using both sets of data separately. To obtain these RC parameters, we fitted a model for the distribution of RC stars in 'true' absolute magnitude, either inferred from an observed absolute magnitude (asteroseismic) or inferred from apparent magnitude, parallax, and extinction (astrometric).

We built a pair of Bayesian hierarchical models with latent parameters that allow us to infer key values such as the distance and the true absolute magnitude from the data and the model. The latent parameters form a stepping stone between our population model, which is described by hyperparameters, and the observations. We use a latent parameter for each star to infer the 'true' distribution of the absolute magnitudes, while fitting our population level model to these inferred 'true' absolute magnitudes, instead of to the observations themselves. Many aspects of our hierarchical models, especially those for the Gaia data, are similar to those used for the same purpose by $\mathrm{H} 17$ with some improvements.

To fit to the position and spread of RC stars while also isolating any outlier contaminants, we applied the mixture model (Hogg et al. 2010) utilised by H17. In this case, we employed two generative models weighted by the mixturemodel weighting factor $Q$. For these we used two normal distributions: one for the inlier population of RC stars, with a mean $\mu_{\mathrm{RC}}$ and a standard deviation (spread) $\sigma_{\mathrm{RC}}$, and a broad outlier distribution centered in the same location $\left(\mu_{\mathrm{RC}}\right)$ but with a spread of $\sigma_{o}$, which must always be larger than $\sigma_{\mathrm{RC}}$. The likelihood to obtain an absolute magnitude $M_{i}$ given this mixture model is then

$$
\begin{aligned}
& p\left(M_{i} \mid \theta_{\mathrm{RC}}\right) \\
& \quad=Q \mathcal{N}\left(M_{i} \mid \mu_{\mathrm{RC}}, \sigma_{\mathrm{RC}}\right)+(1-Q) \mathcal{N}\left(M_{i} \mid \mu_{\mathrm{RC}}, \sigma_{\mathrm{o}}\right),
\end{aligned}
$$

where $M_{i}$ is the true absolute magnitude for a given datum $i, \theta_{R C}=\left\{\mu_{\mathrm{RC}}, \sigma_{\mathrm{RC}}, Q, \sigma_{\mathrm{o}}\right\}$ are the model hyperparameters (which inform the population of latent parameters) and $\mathcal{N}(x \mid \mu, \sigma)$ represents a normal distribution evaluated at $x$, with a mean $\mu$ and a spread $\sigma .^{5}$

\subsection{The asteroseismic model}

For our asteroseismic model, we used a calculated measure of the absolute magnitude $(\hat{M})$ from asteroseismology, along with appropriate uncertainties $\left(\sigma_{\hat{M}}\right)$, as our data. We used a latent variable model to infer the true value of the absolute

5 Note that the spread $\sigma$ as listed in $\mathcal{N}(x \mid \mu, \sigma)$ is not a variance, but a standard deviation, since we are following the nomenclature used in pystan.

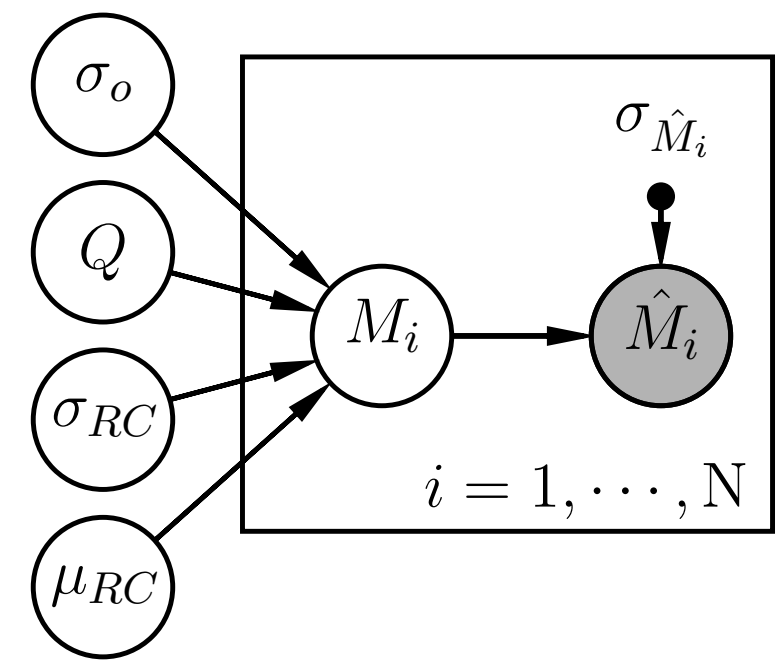

Figure 3. An probabilistic graphical model of the asteroseismic model, represented algebraically in equation 10. Shaded circles indicate observed data, whereas solid black circles represent fixed parameters, such as the uncertainty on the observed data. The hyperparameters $\theta_{\mathrm{RC}}$ can be seen on the left, and inform the set of latent parameters $\boldsymbol{M}_{\boldsymbol{i}}$, which in turn relate to the observed data $\hat{M}_{i}$ and $\sigma_{\hat{M}_{i}}$. N is the number of data points in our sample.

magnitude. Given our data and the hyperparameters on our mixture model $\theta_{\mathrm{RC}}$, we can use Bayes' theorem to find the unnormalised posterior probability of our model:

$p\left(\theta_{\mathrm{RC}} \mid \mathcal{D}\right) \propto p\left(\theta_{\mathrm{RC}}\right) \prod_{i=1}^{\mathrm{N}} p\left(\mathcal{D}_{i} \mid M_{i}\right) p\left(M_{i} \mid \theta_{\mathrm{RC}}\right)$.

Here, $\mathrm{N}$ is the number of points in our data set $\mathcal{D}=\left\{\hat{M}, \sigma_{\hat{M}}\right\}$, $p\left(\mathcal{D}_{i} \mid M_{i}\right)$ is our likelihood function, $p\left(\theta_{\mathrm{RC}}\right)$ represents the priors on the hyperparameters, and $p\left(M_{i} \mid \theta_{\mathrm{RC}}\right)$ is the probability to obtain our latent parameters (the true absolute magnitudes) given our hyperparameters.

The likelihood to obtain our data given our parameters is then

$p\left(\mathcal{D}_{i} \mid M_{i}\right)=\mathcal{N}\left(\hat{M}_{i} \mid M_{i}, \sigma_{\hat{M}_{i}}\right)$,

where $M_{i}$ is the true absolute magnitude. Here, $M_{i}$ is a latent parameter that is drawn from from the likelihood function $p\left(M_{i} \mid \theta_{\mathrm{RC}}\right)$ (equation 9), to which our hyperparameters are fitted. A probabilistic graphical model of the asteroseismic model is shown in Figure 3.

\subsection{The astrometric model}

Fitting the absolute magnitude for the Gaia DR2 sample required a more involved approach, since we wanted to work directly with parallax (Luri et al. 2018). We used a set of three latent parameters, $\alpha_{i}=\left\{M_{i}, r_{i}, A_{i}\right\}$, where $M_{i}$ is the 
absolute magnitude in a given band, $r_{i}$ is the distance and $A_{i}$ is the extinction in a given band. We also include two additional hyperparameters: $\varpi_{\mathrm{zp}}$, the parallax zero-point offset and $L$, the length scale of the exponentially decreasing space density prior on distance (Astraatmadja \& BailerJones 2016a,b, 2017). This prior, which is necessary to treat negative parallax values, has already successfully been applied to Gaia DR2 data (Bailer-Jones et al. 2018) and its use is recommended for this purpose within the Gaia DR2 release papers (Luri et al. 2018).

Some extra care was also required in the treatment of parallax uncertainties for this sample. Lindegren et al. (2018) found parallaxes to be correlated on scales below $40^{\circ}$, with increasing strength at smaller separations, and quantified their covariance using quasar parallaxes. They found the positive covariance $V_{\varpi}$ for these scales to be reasonably approximated by the fitted relation

$V_{\varpi}(\theta) \simeq\left(285 \mu \mathrm{as}^{2}\right) \times \exp \left(-\theta / 14^{\circ}\right)$,

where $\theta$ is the angular separation between two targets in degrees. The fit corresponds to a RMS amplitude of $\sqrt{285 \mu \mathrm{as}^{2}} \approx 17 \mu$ as. This relation was recently applied by Zinn et al. (2018), who found that the Lindegren et al. (2018) relation resulted in the best goodness-of-fit for their models of the parallax zero-point offset, over both a similar relation by Zinn et al. (2017) based on TGAS data, and not including parallax covariances altogether.

We generated a covariance matrix $\Sigma$ for our sample:

$\Sigma_{i j}=V_{\varpi}\left(\theta_{i j}\right)+\delta_{i j} \sigma_{\hat{\varpi}_{i}} \sigma_{\hat{\varpi}_{j}}$,

where $\theta_{i j}$ is the angular separation between stars $i$ and $j$, and $\delta_{i j}$ is the Kronecker delta function.

Given these new additions, our set of data was $\mathcal{D}=$ $\left\{\hat{\varpi}, \underline{\Sigma}, \hat{m}, \sigma_{\hat{m}}, \hat{A}\right\}$, where all symbols are as defined above and $\hat{A}$ is the band specific extinction. We can use Bayes' theorem, as before, to find the unnormalised posterior probability of our model as

$$
\begin{aligned}
& p\left(\theta_{\mathrm{RC}}, \varpi_{\mathrm{zp}}, L, \alpha \mid \mathcal{D}\right) \\
& \quad \propto p\left(\theta_{\mathrm{RC}}, \varpi_{\mathrm{zp}}, L, \alpha\right) p\left(\mathcal{D} \mid \theta_{\mathrm{RC}}, \varpi_{\mathrm{zp}}, L, \alpha\right),
\end{aligned}
$$

where $p\left(\mathcal{D} \mid \theta_{\mathrm{RC}}, \varpi_{\mathrm{zp}}, L, \alpha\right)$ is now our likelihood function and $p\left(\theta_{\mathrm{RC}}, \varpi_{\mathrm{zp}}, L, \alpha\right)$ represents the priors on our hyper- and latent parameters. Our likelihood function relates to two observables as,

$p\left(\mathcal{D} \mid \theta_{\mathrm{RC}}, \varpi_{\mathrm{zp}}, L, \alpha\right)=p\left(\hat{\varpi} \mid r, \varpi_{\mathrm{zp}}, \underline{\Sigma}\right) \times p\left(\hat{m} \mid \alpha, \sigma_{\hat{m}}\right)$.

Note that the parallax only depends on the latent parameter for distance, $r$. Since parallax values are correlated, $p\left(\hat{\varpi} \mid r, \varpi_{\mathrm{zp}}, \underline{\Sigma}\right)$ was evaluated for all data simultaneously, whereas $p\left(\hat{m} \mid \alpha, \sigma_{\hat{m}}\right)$ was evaluated at every datum $i$. This means that our full posterior probability takes the form

$$
\begin{aligned}
& p\left(\theta_{\mathrm{RC}}, \varpi_{\mathrm{zp}}, L, \alpha \mid \mathcal{D}\right) \\
& \quad \propto p\left(\theta_{\mathrm{RC}}, \varpi_{\mathrm{zp}}, L\right) p\left(\hat{\varpi} \mid r, \varpi_{\mathrm{zp}}, \underline{\Sigma}\right) \times \\
& \quad \prod_{i=1}^{\mathrm{N}} p\left(\hat{m}_{i} \mid \alpha_{i}, \sigma_{\hat{m}_{i}}\right) p\left(\alpha_{i} \mid \theta_{\mathrm{RC}}, \varpi_{\mathrm{zp}}, L\right),
\end{aligned}
$$

where the first term represents the priors on our hyperparemeters, the second term is the likelihood to obtain our observed parallaxes, the third is the likelihood to obtain an observed magnitude, and the fourth gives the probability to obtain the latent parameters, given the hyperparameters.

The second component of equation 16 is the probability of obtaining the observed parallax given our latent parameters and our covariance matrix. Since we treated our parallax uncertainties as correlated, we evaluated these probabilities for the full set using a multivariate normal distribution:

$p\left(\hat{\varpi} \mid r, \varpi_{\mathrm{zp}}, \underline{\Sigma}\right)=\mathcal{N}\left(\hat{\varpi} \mid 1 / r+\varpi_{\mathrm{zp}}, \underline{\Sigma}\right)$,

where $1 / r$ defines the true parallax. The latent parameters for the distance $r_{i}$ were drawn from an exponentially decreasing space density prior (Bailer-Jones 2015), which goes as

$p\left(r_{i} \mid L\right)=\frac{1}{2 L^{3}} r_{i}^{2} \exp \left(-r_{i} / L\right)$,

and thus depends on the length scale hyperparameter $L$. This prior has a mode at $2 L$, beyond which it decreases exponentially.

The third component of equation 16 is then

$p\left(\hat{m}_{i} \mid \alpha_{i}, \sigma_{\hat{m}_{i}}\right)=\mathcal{N}\left(\hat{m}_{i} \mid m_{i}, \sigma_{\hat{m}_{i}}\right)$,

where $m_{i}$ is the true apparent magnitude, and is drawn from the relation

$m_{i}=M_{i}+5 \log _{10}\left(r_{i}\right)-5+A_{i}$.

Here, we have used the inferred true values for absolute magnitude, distance and extinction to calculate apparent magnitude. As for the seismic method, the true absolute magnitude $M_{i}$ was drawn from the likelihood $p\left(M_{i} \mid \theta_{R C}\right)$, as given in equation 9 . The final latent parameter $A_{i}$ is given a prior as

$p\left(A_{i} \mid \hat{A}_{i}\right)=\mathcal{N}\left(A_{i} \mid \hat{A}_{i}, 0.05\right)$,

a normal distribution with a spread of $0.05 \mathrm{mag}$, where $\hat{A}_{i}$ is our observed value for the extinction (Green et al. 2018). A probabilistic graphical model of the astrometric model is shown in Figure 4.

\subsection{Priors on the hyperparameters}

The priors on the hyperparameters were, where possible, identical across both models. For the asteroseismic model, our priors took the form of

$$
\begin{aligned}
\mu_{\mathrm{RC}} & \sim \mathcal{N}\left(\mu_{\mathrm{H}}, 1\right) \\
\sigma_{\mathrm{RC}} & \sim \mathcal{N}(0,1) \\
Q & \sim \mathcal{N}(1,0.25) \\
\sigma_{\mathrm{o}} & \sim \mathcal{N}(3,2),
\end{aligned}
$$

where $\mu_{\mathrm{H}}$ is the absolute magnitude of the $\mathrm{RC}$ in the relevant passband, as reported by $\mathrm{H} 17$, and $\sigma_{\mathrm{RC}}$ must be above 0 . It 


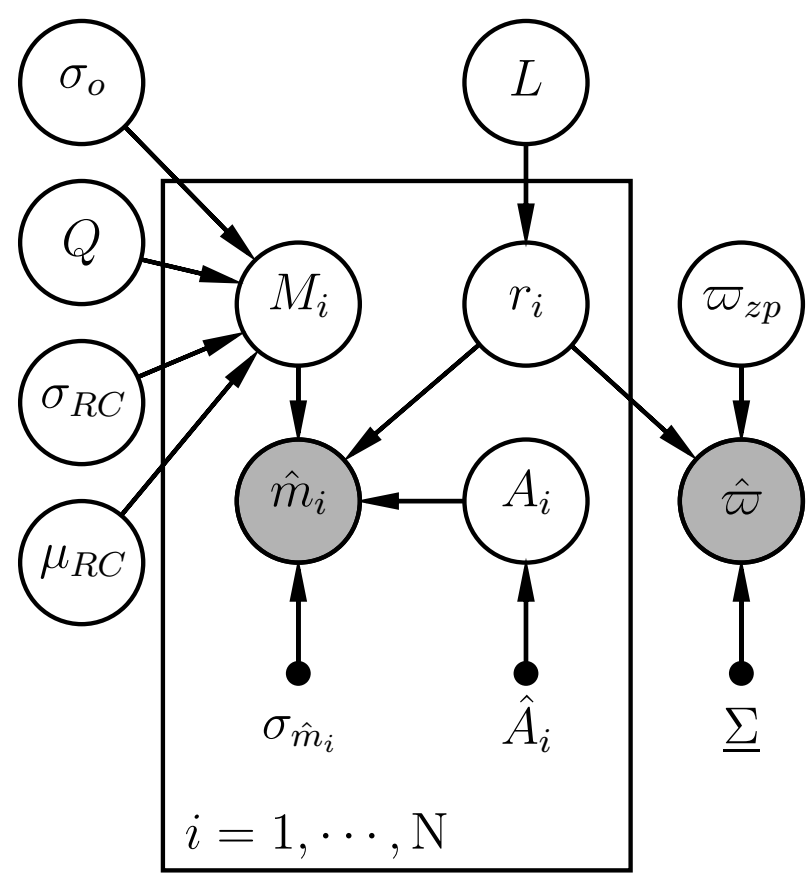

Figure 4. An acyclic diagram of the astrometric model, represented algebraically in equation 16 . Conventions are the same as for Figure 3. The full parallax covariance matrix is denoted as $\underline{\Sigma}$; it should be noted that the parallax likelihood is evaluated across the full set as a multivariate normal distribution.

should be noted that, in order to evaluate the hierarchical mixture model in PyStan, $\sigma_{\mathrm{o}}$ is expressed in units of $\sigma_{\mathrm{RC}}$ and must always be larger than 1 to ensure the two components of the mixture model do not switch roles. $Q$ must fall within the range 0.5 to 1 , because we expect an inlier-dominated sample.

For the astrometric method, we introduced the two new parameters $\varpi_{\mathrm{zp}}$ and $L$, and applied a new prior to $\mu_{\mathrm{RC}}$ and $\sigma_{\mathrm{RC}}$, while the priors for the other hyperparameters remained the same:

$$
\begin{aligned}
\mu_{\mathrm{RC}} & \sim \mathcal{N}\left(\mu_{\mathrm{RC}, \text { seis }}, \sigma_{\mu_{\mathrm{RC}, \text { seis }}}\right) \\
\sigma_{\mathrm{RC}} & \sim \mathcal{N}\left(\sigma_{\mathrm{RC}, \text { seis }}, \sigma_{\sigma_{\mathrm{RC}, \text { seis }}}\right) \\
L & \sim \mathcal{U}(0.1,4000) \\
\varpi_{\mathrm{zp}} & \sim \mathcal{N}(0,500) .
\end{aligned}
$$

Here, $\mathcal{U}$ denotes a uniform distribution with the lower and upper limits as arguments, and the units of $\varpi_{\mathrm{zp}}$ and $L$ are $\mu$ as and kpc, respectively. The quantities $\mu_{\mathrm{RC} \text {,seis }}$ and $\sigma_{\mathrm{RC} \text {, seis }}$ are the medians of the posterior distributions on $\mu_{\mathrm{RC}}$ and $\sigma_{\mathrm{RC}}$ from the asteroseismic model, and $\sigma_{\mu_{\mathrm{RC} \text {, seis }}}$ and $\sigma_{\sigma_{\mathrm{RC} \text {, seis }}}$ are the spreads on the posteriors, effectively allowing us to explore what value of the parallax-zero point offset, $\varpi_{\mathrm{zp}}$, recovers the results we see using asteroseismology.

Finally, for runs where we investigated the impact of literature values for $\varpi_{\mathrm{zp}}$ on our RC parameters, we set the priors on $\mu_{\mathrm{RC}}$ and $\sigma_{\mathrm{RC}}$ to those used on our seismic run, and applied a prior on $\varpi_{\mathrm{zp}}$ as

$\varpi_{\mathrm{zp}} \sim \mathcal{N}\left(\varpi_{\mathrm{zp}, \text { lit }}, \sigma_{\varpi_{\mathrm{zp}, \mathrm{lit}}}\right)$.

Here, $\varpi_{\mathrm{zp}, \text { lit }}$ and $\sigma_{\varpi_{\mathrm{zp}, \text { lit }}}$ are values and uncertainties on said values from the literature.

We drew samples from the posterior distributions using PyStan version 2.18.0.0, with four chains and 5000 iterations, with half of the iterations used as burn-in. Appropriate convergence of our chains was evaluated using the $\hat{R}$ diagnostic. ${ }^{6}$

\section{RESULTS}

\subsection{Results from asteroseismology}

To see how the absolute magnitude $\mu_{\mathrm{RC}}$ and spread $\sigma_{\mathrm{RC}}$ of the $\mathrm{RC}$ change given our input data, we applied two changes to calculations for seismic absolute magnitude. First, we perturbed the temperature by a value $\Delta T_{\text {eff }}$ that ranged between -50 and $50 \mathrm{~K}$, in steps of $10 \mathrm{~K}$. Second, we propagated these temperatures, along with the original and unperturbed uncertainties on $T_{\mathrm{eff}}, v_{\max }$ and $\Delta v$, through the seismic scaling relations to find luminosity. We did this both with and without calibrations for the $\Delta v$ scaling relation obtained by the grid interpolation method by Sharma et al. (2016). The perturbed temperatures were also used in the grid interpolation required to obtain the correction (Sharma \& Stello 2016), and the corrections were thus recalculated for each change in temperature. We also calculated BCs for each set of temperatures, and recalculated a seismic $\log g$ given the perturbed temperatures for each calculation of the BCs (Casagrande \& VandenBerg 2014, 2018a,b). Seismic radii were calculated per equation 5 , which were in turn used to calculate luminosities and were combined with the BCs to compute our absolute magnitudes, resulting in 22 individual sets that differ in corrections to the seismic scaling relations and temperature scale, for both photometric bands.

Our results for our Y18 sample are shown in Tables 1 \& 3 where we present the medians of the posterior distributions for our hyperparameters for the 2MASS $K$ band and Gaia $G$ band respectively, both with and without a correction to the $\Delta v$ scaling relation, for various changes in temperature scale. Uncertainties are given as the $1 \sigma$ credible intervals. Where the posterior distributions are approximately Gaussian we quote a symmetric single uncertainty. The change of the posterior on the magnitude of the $\mathrm{RC} \mu_{\mathrm{RC}}$ alone, given the input, can be seen in Figure 5.

For our APOKASC-2 temperature subsample of 1637 stars, we reran our models using the same methodology as before, simply substituting the temperatures and temperature uncertainties reported in Pinsonneault et al. (2018) for those in Y18 for those stars, and making no other changes. Note that the change in temperature values carried through to the calculation of the bolometric corrections and corrections to the scaling relations for each run. The results of this

${ }^{6}$ Our code is open and can be found on Github at https://www. github.com/ojhall94/halletal2019 
are presented in Tables 2 and 4 for all hyperparameters, as with the run on the full sample. The change in the posteriors on the position of the RC is shown for this reduced sample in Figure 6.

\subsection{Results from Gaia}

Given our results from asteroseismology, we wish to determine the parallax zero-point offset, $\varpi_{\mathrm{zp}}$, that recovers our values of the absolute magnitude and spread of the RC. Since $\mu_{\mathrm{RC}}$ and $\sigma_{\mathrm{RC}}$ represent astrophysical observables that should be consistent across both data sets, we used a description of the posterior distributions from these parameters from our seismic model as a highly informative prior in our Gaia model. This yields the parallax offset required to recover the same magnitude and spread of the RC found using seismology. We passed in the seismic posteriors for $\Delta T_{\text {eff }}$ being -50 , 0 , and $+50 \mathrm{~K}$ from our runs on our full sample and the reduced APOKASC-2 sample, and thus ran our model for 6 different RC magnitudes \& spreads in each band. Additionally, we used the median values of each latent parameter $M_{i}$ from the application of our seismic model to our full sample, along with distance estimates by Bailer-Jones et al. (2018) and observed extinctions from Green et al. (2018), as initial guesses in our Gaia model for computational efficiency. No other values were changed on each run.

Following the relation presented in equation 12 (Lindegren et al. 2018) we treated our parallax uncertainties as correlated as a function of position on the sky across the entire Kepler field, similarly to previous work by Zinn et al. (2018). While the model equation presented by Lindegren et al. (2018) describes the covariance well for a wide range of separations, individual covariances oscillate around the model at separations below $1 \mathrm{deg}$, and the model no longer holds at all for separations below $0.125 \mathrm{deg}$. To ensure that our treatment of the parallax covariances was sensible, we ran our Gaia model on a reduced sample of 1000 stars, randomly selected from across the entire Kepler field to ensure sparsity. This reduced sample contained no angular separations in the range $<0.125 \mathrm{deg}^{7}$.

In Tables 5, 6, 7 and 8 we present the medians on the posterior distributions of our hyperparameters for our Gaia model, given RC-corrected seismic positions and spreads for the RC at different temperature offsets $\Delta T_{\text {eff }}$ for both the Y18 and APOKASC-2 samples. In Figure 7, we present the posterior distributions of $\varpi_{\mathrm{zp}}$ given the 6 values for the position of the RC used each in the $K$ and $G$ bands.

In order to probe the impact of literature values for $\varpi_{\mathrm{zp}}$ on an inference of our RC parameters, we reran our Gaia model for the $K$ and Gaia $G$ bands with a strongly informative prior on $\varpi_{\mathrm{zp}}$ (see equation 24). We did this for the same reduced sample of 1000 stars from our Y18 sample. For all these runs, we applied the same priors used for $\mu_{\mathrm{RC}}$ and $\sigma_{\mathrm{RC}}$ as in the asteroseismic runs (see equation 22). We used the parallax zero-point offsets reported by Lindegren et al. (2018) ( $-29 \mu$ as, with an assumed uncertainty of $1 \mu$ as $)$, Zinn et al. (2018) (-52.8 $\mu$ as with a total uncertainty of 3.4

7 The data were shuffled using the sklearn.utils.shuffle function with a random seed of 24601 . $\mu$ as), Riess et al. (2018) $(-46 \pm 13 \mu$ as $)$, Sahlholdt \& Silva Aguirre (2018) (-35 $\pm 16 \mu$ as) and Stassun \& Torres (2018) $(-82 \pm 33 \mu$ as $)$. Note that for the purpose of calibration not all these zero-point offsets would be applicable to our sample due to differences in colour, magnitude, and position. We instead used them as representative of $\varpi_{\mathrm{zp}}$ in the literature to study their impact on our inferences only. In addition, we also ran with a prior of $0 \pm 1 \mu$ as in an attempt to recreate the H17 work (albeit accounting for parallax covariances), as well as a single run with no strongly informative priors on $\varpi_{\mathrm{zp}}, \mu_{\mathrm{RC}}$ or $\sigma_{\mathrm{RC}}$, thus finding our own measure of the zero-point offset.

In Tables $9 \& 10$ we present the medians and $1 \sigma$ credible intervals on the posterior distributions for the hyperparameters of our Gaia model given the conditions stated above, as well as naming the source of the used parallax zero-point offset, and an expression of the prior applied to $\varpi_{\mathrm{zp}}$. Note that the inferred value of $\varpi_{\mathrm{zp}}$ may differ significantly within the uncertainties on any of the literature values used. In Figure 8 we present the medians and $1 \sigma$ credible intervals on the posterior distributions for $\mu_{\mathrm{RC}}$ given our chosen values for $\varpi_{\mathrm{zp}}$, with the result from the 'uninformed' run shown with bold red error bars.

\section{DISCUSSION}

\subsection{Luminosity of the Red Clump}

Figures 5 and 6 show the posteriors on the inferred absolute magnitude of the $\mathrm{RC}, \mu_{\mathrm{RC}}$, for the $K$ and Gaia $G$ bands given changes to effective temperature and corrections to the scaling relations. There is a clear relation between the overall offset in $T_{\text {eff }}$ and the inferred magnitude of the RC, where a change of about $20 \mathrm{~K}$ results in a difference of more than $1 \sigma$. The overall relation between the clump magnitude and temperature is expected, given the large impact of temperature on the calculations for absolute magnitude; luminosity calculated via the seismic scaling relations scales with temperature to a power of 4.5, and bolometric corrections calculated through the Casagrande \& VandenBerg (2018b) method rely on both $T_{\text {eff }}$ and $\log g$, which is calculated using $T_{\text {eff. }}$. The small uncertainties on $\mu_{\mathrm{RC}}$ and $\sigma_{\mathrm{RC}}$ indicate the ability of hierarchical models to leverage a large number of individual uncertainties to fit to a population model, given that the uncertainties on our data for $T_{\text {eff }}$ are well above the shifts in temperature we are applying.

We also see that the scaling relation corrections appear to be degenerate with a small temperature offset. A change of $\sim 20 \mathrm{~K}$ to the temperatures provides a similar clump magnitude as when applying a correction to the scaling relations. At higher temperatures, the difference in the magnitude of the RC between corrected and uncorrected scaling relations increases. This shows that the $T_{\text {eff }}$ values have a significant impact on the $f_{\Delta v}$ obtained through the Sharma \& Stello (2016) method, even at relatively small $T_{\text {eff }}$ shifts.

The values for $\mu_{\mathrm{RC}}$ in both bands are fainter for the subset of stars using APOKASC-2 temperatures than those using temperatures from Mathur et al. (2017). This reflects the relation we already saw between $T_{\text {eff }}$ and $\mu_{\mathrm{RC}}$ for the Y18 stars, since the stars in the APOKASC-2 subsample represent a population subset of lower-temperature stars, as well 

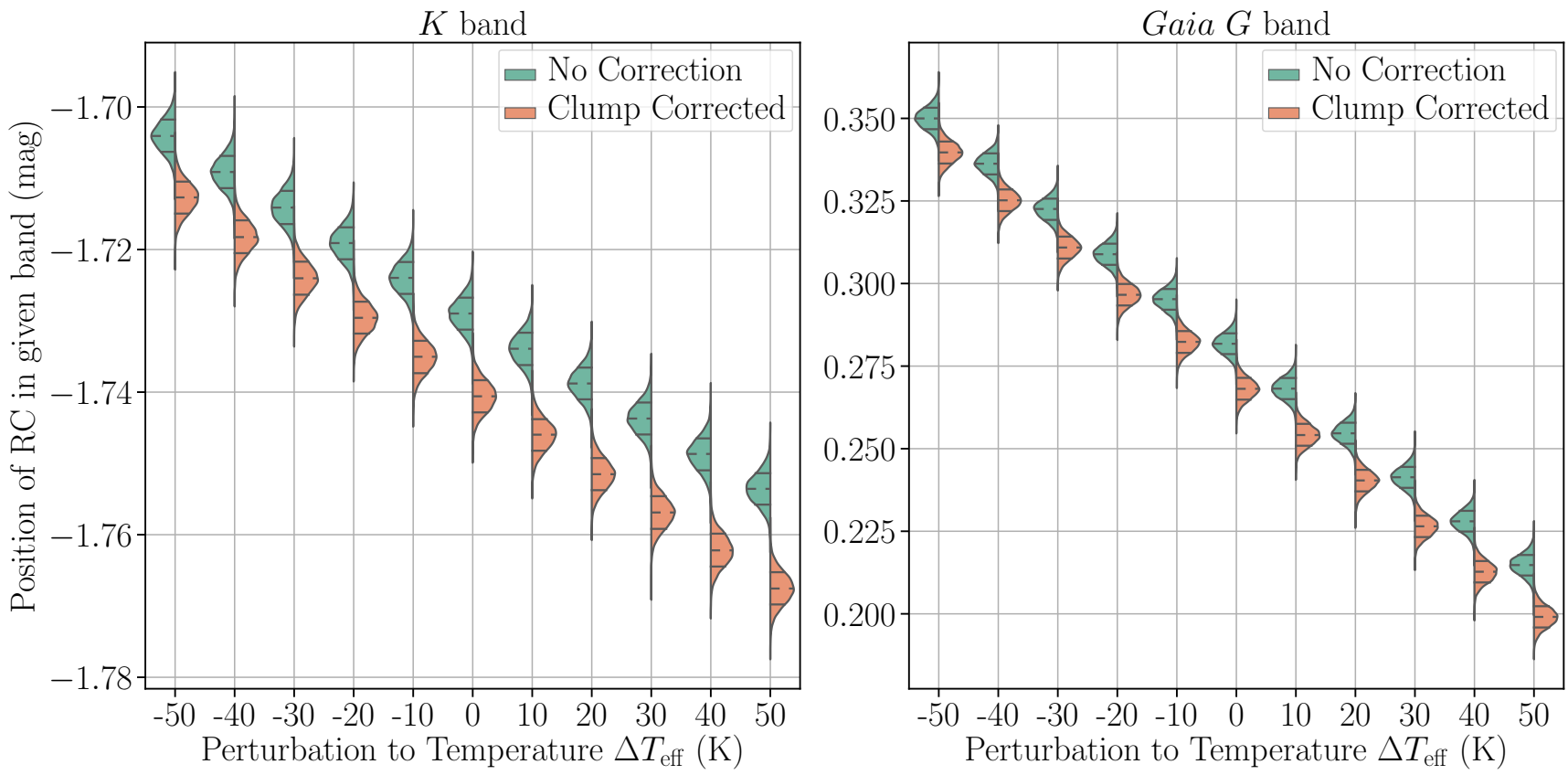

Figure 5. The posterior distributions on the position of the Red Clump in the 2MASS $K$ band (left) and Gaia $G$ band (right), as a function of overall perturbation to the temperature values $\Delta T_{\text {eff }}$ using asteroseismology, both with (orange) and without (green) corrections to the $\Delta v$ scaling relation (Sharma et al. 2016). The dashed horizontal lines indicate the median on the posteriors, and the solid horizontal lines represent the $1 \sigma$ credible intervals. The posteriors' magnitudes along the x-axis are indicative of power with arbitrary units, whereas their shape along the $\mathrm{y}$-axis indicates the spread in the posterior result.

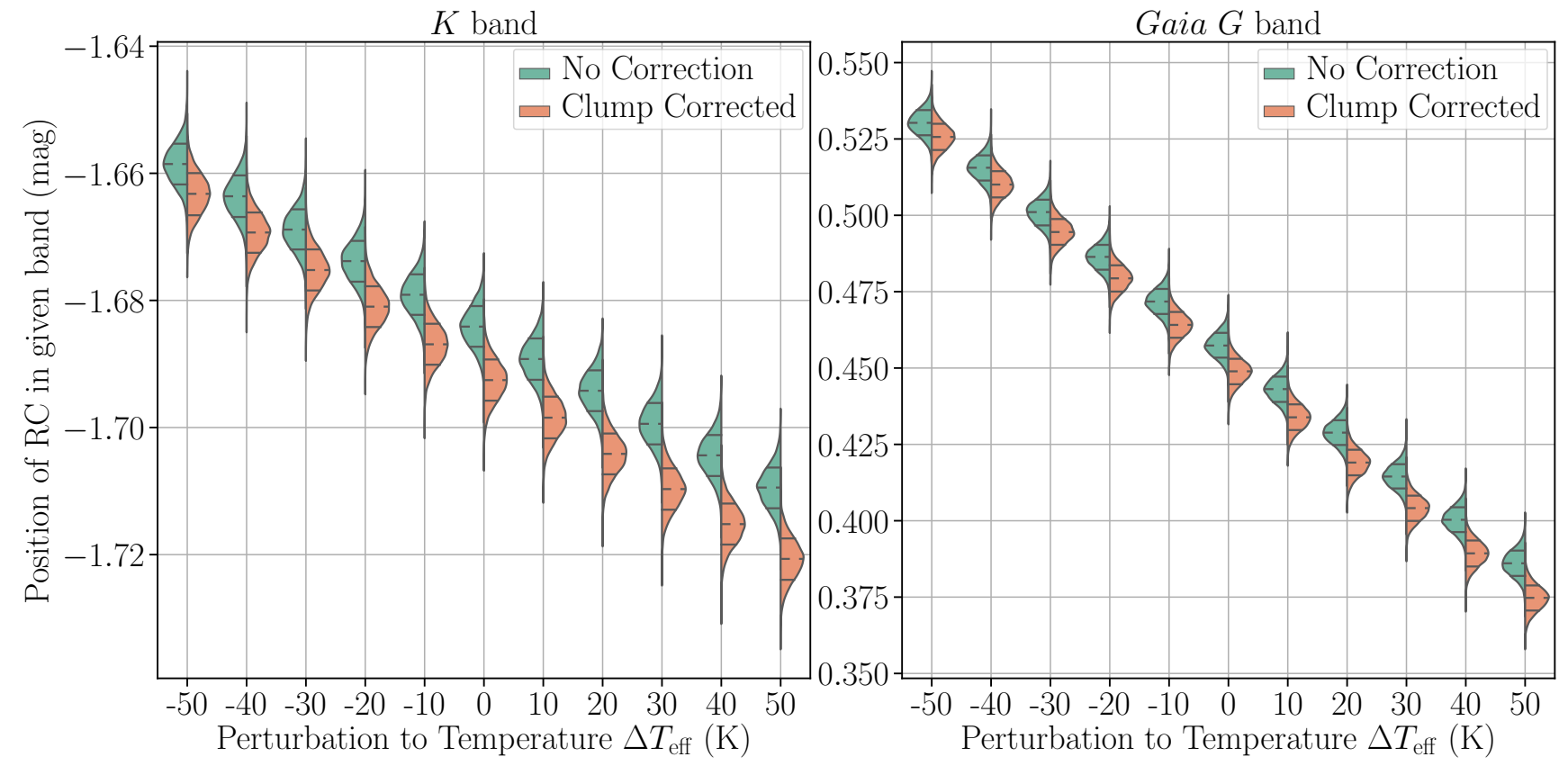

Figure 6. The same as Figure 5, except using only stars both in our sample and the APOKASC-2 (Pinsonneault et al. 2018) sample, replacing $T_{\text {eff }}$ with those reported in APOKASC-2. 

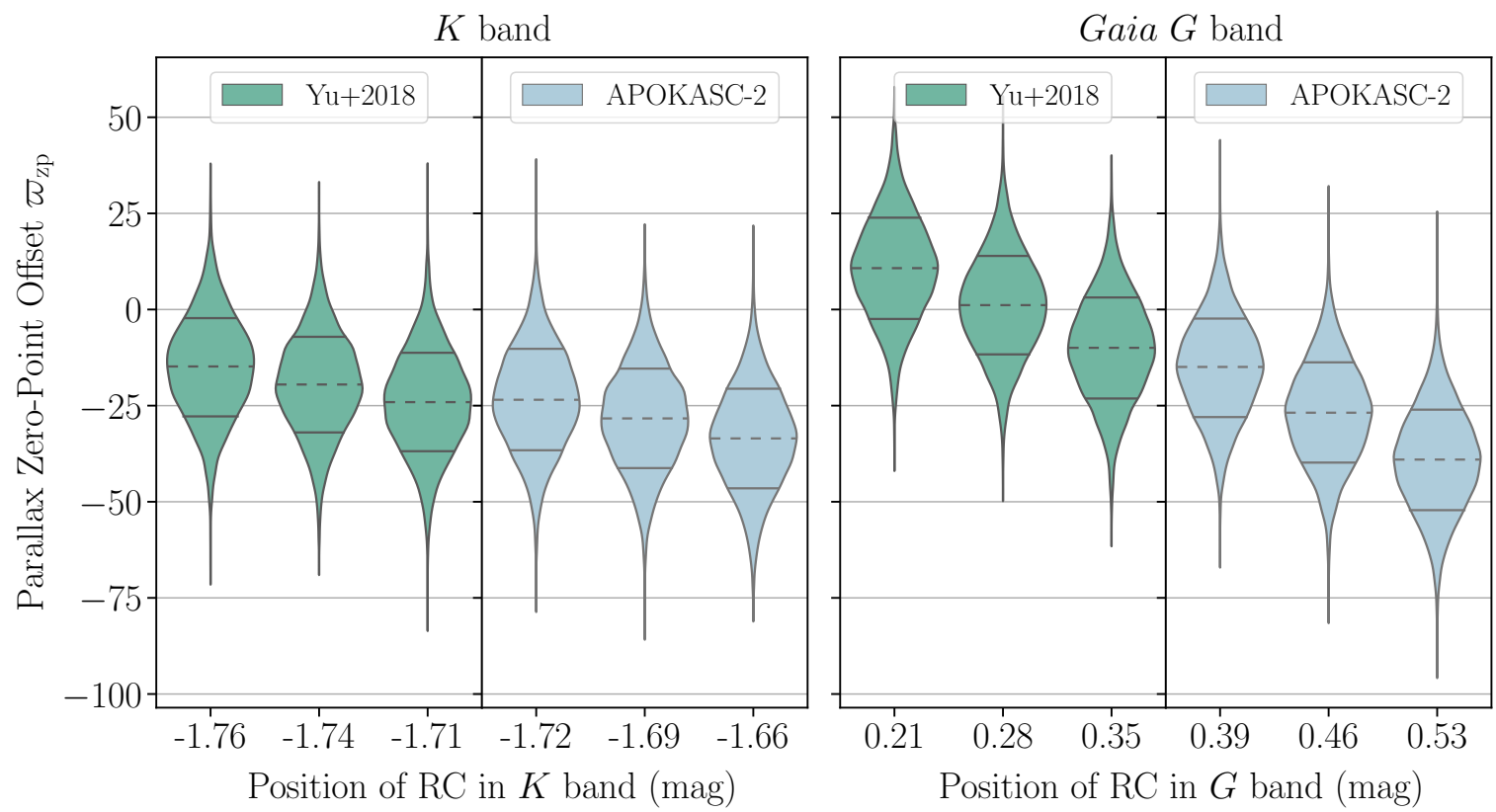

Figure 7. The posterior distributions on the parallax zero-point offset $\varpi_{\text {zp }}$, as a function of the absolute magnitude of the RC used to calibrate this value, for 1000 randomly selected stars across the Kepler field. The RC magnitudes on the $\mathrm{x}$-axis correspond to those obtained from seismology for perturbations to the temperature values $\Delta T_{\text {eff }}$ of $-50,0$, and $+50 \mathrm{~K}$, from runs on our full sample (Yu et al. 2018) and the APOKASC-2 sample (Pinsonneault et al. 2018). The dashed horizontal lines indicate the median on the posteriors, and the solid horizontal lines represent the $1 \sigma$ credible intervals. The posteriors' magnitudes along the x-axis are indicative of power with arbitrary units, whereas their shape along the $\mathrm{y}$-axis indicates the spread in the posterior result, and is reflected across the $\mathrm{x}$-axis.

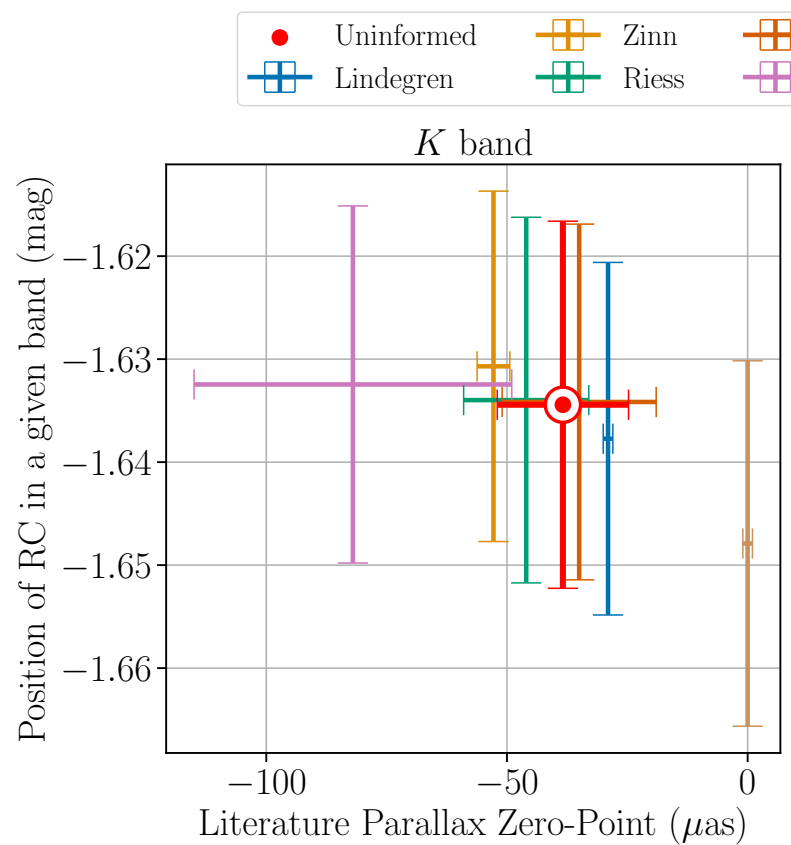

Sahlholdt \& Silva Aguirre $\square \square$ Hawkins
Stassun \& Torres

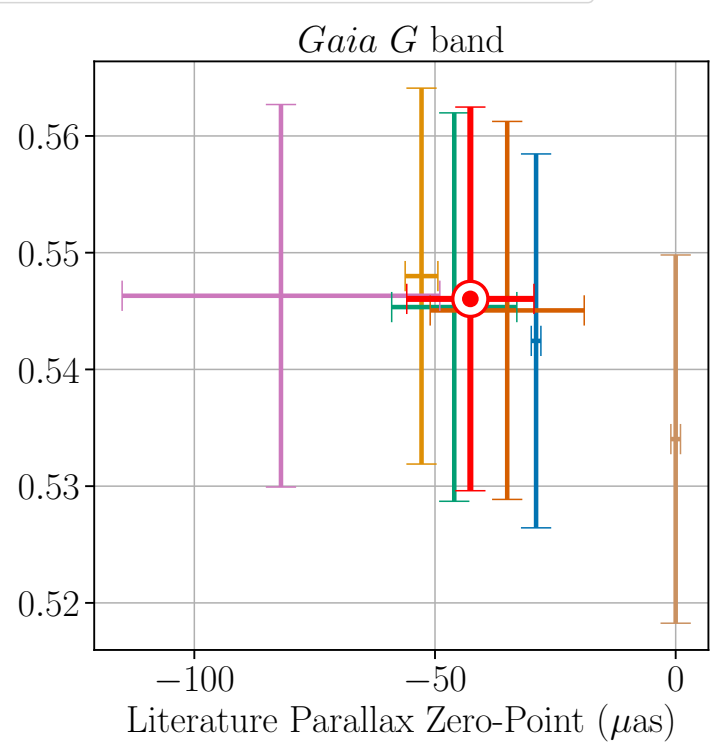

Figure 8. The the $1 \sigma$ credible intervals for the posterior distributions on $\mu_{\mathrm{RC}}$, as a function of the value for $\varpi_{\mathrm{zp}}$ used as an informative prior on $\varpi_{\mathrm{zp}}$, for 1000 randomly selected stars across the Kepler field in both the 2MASS $K$ and $G a i a G$ bands. The errorbars on the $\mathrm{x}$-axis correspond to the formal uncertainties for literature values, or are otherwise specified in the text. the 'uninformed' value corresponds to a run of our Gaia model with no strong constrains on $\varpi_{\mathrm{zp}}$, and in this case the x-axis errobars correspond go the $1 \sigma$ credible intervals on the inferred value for $\varpi_{\mathrm{zp}}$. 


\begin{tabular}{|c|c|c|c|c|c|c|c|c|}
\hline \multirow[b]{2}{*}{$\Delta T_{\text {eff }}(K)$} & \multicolumn{4}{|c|}{ No Correction } & \multicolumn{4}{|c|}{ Clump Corrected } \\
\hline & $\mu_{\mathrm{RC}}(\mathrm{mag})$ & $\sigma_{\mathrm{RC}}(\mathrm{mag})$ & $Q$ & $\sigma_{\mathrm{o}}\left(\sigma_{\mathrm{RC}}\right)$ & $\mu_{\mathrm{RC}}(\mathrm{mag})$ & $\sigma_{\mathrm{RC}}(\mathrm{mag})$ & $Q$ & $\sigma_{\mathrm{o}}\left(\sigma_{\mathrm{RC}}\right)$ \\
\hline-50.0 & $-1.704 \pm 0.002$ & $0.03 \pm 0.003$ & $0.92 \pm 0.01$ & $10.35_{-1.01}^{+1.17}$ & $-1.713 \pm 0.002$ & $0.034 \pm 0.004$ & $0.91 \pm 0.01$ & $8.85_{-0.93}^{+1.09}$ \\
\hline-40.0 & $-1.709 \pm 0.002$ & $0.03 \pm 0.003$ & $0.92 \pm 0.01$ & $10.33_{-1.01}^{+1.01}$ & $-1.718 \pm 0.002$ & $0.033 \pm 0.004$ & $0.91 \pm 0.01$ & $9.11_{-1.04}^{+1.93}$ \\
\hline-30.0 & $-1.714 \pm 0.002$ & $0.03 \pm 0.003$ & $0.92 \pm 0.01$ & $10.4_{-1.04}^{+1.015}$ & $-1.724 \pm 0.002$ & $0.033 \pm 0.004$ & $0.91 \pm 0.01$ & $9.16_{-0.96}^{+1.12}$ \\
\hline-20.0 & $-1.719 \pm 0.002$ & $0.029 \pm 0.003$ & $0.92 \pm 0.01$ & $10.55_{-1.05}^{+1.04}$ & $-1.73 \pm 0.002$ & $0.033 \pm 0.004$ & $0.91 \pm 0.01$ & $9.22_{-0.91}^{+1.06}$ \\
\hline-10.0 & $-1.724 \pm 0.002$ & $0.03 \pm 0.003$ & $0.92 \pm 0.01$ & $10.49_{-1.03}^{+1.13}$ & $-1.735 \pm 0.002$ & $0.033 \pm 0.004$ & $0.91 \pm 0.01$ & $9.16_{-0.98}^{+1.09}$ \\
\hline 0.0 & $-1.729 \pm 0.002$ & $0.03 \pm 0.003$ & $0.92 \pm 0.01$ & $10.33_{-1.01}^{+1.19}$ & $-1.741 \pm 0.002$ & $0.033 \pm 0.004$ & $0.91 \pm 0.01$ & $9.18_{-0.92}^{+1: 09}$ \\
\hline 10.0 & $-1.734 \pm 0.002$ & $0.029 \pm 0.003$ & $0.92 \pm 0.01$ & $10.44_{-0.97}^{+1.01}$ & $-1.746 \pm 0.002$ & $0.032 \pm 0.004$ & $0.91 \pm 0.01$ & $9.36_{-1.05}^{+1: 2^{2}}$ \\
\hline 20.0 & $-1.739 \pm 0.002$ & $0.03 \pm 0.004$ & $0.92 \pm 0.01$ & $10.32_{-1.02}^{+1.17}$ & $-1.752 \pm 0.002$ & $0.033 \pm 0.004$ & $0.91 \pm 0.01$ & $9.19_{-1.01}^{+1.16}$ \\
\hline 30.0 & $-1.744 \pm 0.002$ & $0.03 \pm 0.003$ & $0.92 \pm 0.01$ & $10.41_{-0.99}^{+1.06}$ & $-1.757 \pm 0.002$ & $0.032 \pm 0.004$ & $0.91 \pm 0.01$ & $9.37_{-1.02}^{+1.16}$ \\
\hline 40.0 & $-1.749 \pm 0.002$ & $0.03 \pm 0.003$ & $0.92 \pm 0.01$ & $10.41_{-1.02}^{+1.18}$ & $-1.762 \pm 0.002$ & $0.032 \pm 0.004$ & $0.91 \pm 0.01$ & $9.37_{-0.97}^{+1.02}$ \\
\hline 50.0 & $-1.754 \pm 0.002$ & $0.03 \pm 0.003$ & $0.92 \pm 0.01$ & $10.27_{-1.01}^{+1.12}$ & $-1.768 \pm 0.002$ & $0.032 \pm 0.004$ & $0.91 \pm 0.01$ & $9.25_{-1}^{+1.16}$ \\
\hline
\end{tabular}

Table 1. Medians of the posterior distributions for hyperparameters of our seismic model, for the 2MASS $K$ band, for 5576 stars from the Y18 sample. Uncertainties are taken as the $1 \sigma$ credible intervals, and are listed as a single value for cases where the posterior was approximately Gaussian. Values are listed for data that have been left uncorrected (No Correction) and data with an appropriate correction to the seismic scaling relations (Clump Corrected). $\Delta T_{\text {eff }}$ is the global shift to our values of $T_{\mathrm{eff}}, \mu_{\mathrm{RC}}$ is the position of the RC in absolute magnitude, $\sigma_{\mathrm{RC}}$ is the spread of the RC in absolute magnitude, $Q$ is the mixture model weighting factor (and the effective fraction of stars considered inliers), and $\sigma_{o}$ is the spread of our outlier population, expressed in terms of $\sigma_{\mathrm{RC}}$.

\begin{tabular}{|c|c|c|c|c|c|c|c|c|}
\hline \multirow[b]{2}{*}{$\Delta T_{\text {eff }}(K)$} & \multicolumn{4}{|c|}{ No Correction } & \multicolumn{4}{|c|}{ Clump Corrected } \\
\hline & $\mu_{\mathrm{RC}}(\mathrm{mag})$ & $\sigma_{\mathrm{RC}}(\mathrm{mag})$ & $Q$ & $\sigma_{\mathrm{o}}\left(\sigma_{\mathrm{RC}}\right)$ & $\mu_{\mathrm{RC}}(\mathrm{mag})$ & $\sigma_{\mathrm{RC}}(\mathrm{mag})$ & $Q$ & $\sigma_{\mathrm{o}}\left(\sigma_{\mathrm{RC}}\right)$ \\
\hline-50.0 & $-1.659 \pm 0.003$ & $0.029 \pm 0.004$ & $0.9 \pm 0.02$ & $9.2_{-1.09}^{+1.2}$ & $-1.663 \pm 0.003$ & $0.031 \pm 0.005$ & $0.89 \pm 0.02$ & $8.46_{-1.06}^{+1.19}$ \\
\hline-40.0 & $-1.664 \pm 0.003$ & $0.029 \pm 0.004$ & $0.9 \pm 0.02$ & $9.14_{-1.08}^{+1.18}$ & $-1.669 \pm 0.003$ & $0.032 \pm 0.005$ & $0.89 \pm 0.02$ & $8.4_{-1.1}^{+1.06}$ \\
\hline-30.0 & $-1.669 \pm 0.003$ & $0.029 \pm 0.004$ & $0.9 \pm 0.02$ & $9.13_{-1.11}^{+1.23}$ & $-1.675 \pm 0.003$ & $0.031 \pm 0.005$ & $0.89 \pm 0.02$ & $8.53_{-1.06}^{+1.16}$ \\
\hline-20.0 & $-1.674 \pm 0.003$ & $0.029 \pm 0.004$ & $0.9 \pm 0.02$ & $9.15_{-1.1}^{+1.26}$ & $-1.681 \pm 0.003$ & $0.031 \pm 0.005$ & $0.89 \pm 0.02$ & $8.43_{-1.06}^{+1.09}$ \\
\hline-10.0 & $-1.679 \pm 0.003$ & $0.03 \pm 0.004$ & $0.9 \pm 0.02$ & $9.11_{-1.09}^{+1.18}$ & $-1.687 \pm 0.003$ & $0.032 \pm 0.005$ & $0.89 \pm 0.02$ & $8.37_{-1.11}^{+1.02}$ \\
\hline 0.0 & $-1.684 \pm 0.003$ & $0.029 \pm 0.004$ & $0.9 \pm 0.02$ & $9.13_{-1.1}^{+1.25}$ & $-1.693 \pm 0.003$ & $0.031 \pm 0.005$ & $0.89 \pm 0.02$ & $8.5_{-1.08}^{+1.118}$ \\
\hline 10.0 & $-1.689 \pm 0.003$ & $0.03 \pm 0.004$ & $0.9 \pm 0.02$ & $9.08_{-1.28}^{+1.1}$ & $-1.698 \pm 0.003$ & $0.032 \pm 0.005$ & $0.89 \pm 0.02$ & $8.41_{-1.06}^{+1.08}$ \\
\hline 20.0 & $-1.694 \pm 0.003$ & $0.03 \pm 0.004$ & $0.9 \pm 0.02$ & $9.04_{-1.06}^{+1.08}$ & $-1.704 \pm 0.003$ & $0.032 \pm 0.005$ & $0.89 \pm 0.02$ & $8.44_{-1.08}^{+1.06}$ \\
\hline 30.0 & $-1.699 \pm 0.003$ & $0.029 \pm 0.004$ & $0.9 \pm 0.02$ & $9.1_{-1.17}^{+1.06}$ & $-1.71 \pm 0.003$ & $0.033 \pm 0.005$ & $0.9 \pm 0.02$ & $8.29^{+1.21}$ \\
\hline 40.0 & $-1.704 \pm 0.003$ & $0.029 \pm 0.004$ & $0.9 \pm 0.02$ & $9.12_{-1.1}^{+1.018}$ & $-1.715 \pm 0.003$ & $0.032 \pm 0.005$ & $0.89 \pm 0.02$ & $8.43_{-1.07}^{+1.23}$ \\
\hline 50.0 & $-1.709 \pm 0.003$ & $0.029 \pm 0.004$ & $0.9 \pm 0.02$ & $9.15_{-1.09}^{+1.24}$ & $-1.721 \pm 0.003$ & $0.032 \pm 0.005$ & $0.89 \pm 0.02$ & $8.39_{-1.07}^{+1.19}$ \\
\hline
\end{tabular}

Table 2. Same as Table 1, except for a subsample of stars from the APOKASC-2 (Pinsonneault et al. 2018) sample.

\begin{tabular}{|c|c|c|c|c|c|c|c|c|}
\hline \multirow[b]{2}{*}{$\Delta T_{\text {eff }}(K)$} & \multicolumn{4}{|c|}{ No Correction } & \multicolumn{4}{|c|}{ Clump Corrected } \\
\hline & $\mu_{\mathrm{RC}}(\mathrm{mag})$ & $\sigma_{\mathrm{RC}}(\mathrm{mag})$ & $Q$ & $\sigma_{\mathrm{o}}\left(\sigma_{\mathrm{RC}}\right)$ & $\mu_{\mathrm{RC}}(\mathrm{mag})$ & $\sigma_{\mathrm{RC}}(\mathrm{mag})$ & $Q$ & $\sigma_{\mathrm{o}}\left(\sigma_{\mathrm{RC}}\right)$ \\
\hline-50.0 & $0.35 \pm 0.003$ & $0.181 \pm 0.004$ & $0.98 \pm 0.01$ & $2.73_{-0.42}^{+0.58}$ & $0.34 \pm 0.003$ & $0.193 \pm 0.004$ & $0.99 \pm 0.01$ & $2.77_{-0.46}^{+0.66}$ \\
\hline-40.0 & $0.336 \pm 0.003$ & $0.181 \pm 0.004$ & $0.98 \pm 0.01$ & $2.73_{-0.4}^{+0.45}$ & $0.325 \pm 0.003$ & $0.192 \pm 0.004$ & $0.99 \pm 0.01$ & $2.78_{-0.45}^{+0.66}$ \\
\hline-30.0 & $0.323 \pm 0.003$ & $0.18 \pm 0.004$ & $0.98 \pm 0.01$ & $2.72_{-0.04}^{+0.4}$ & $0.311 \pm 0.003$ & $0.19 \pm 0.004$ & $0.99 \pm 0.01$ & $2.79_{-0.43}^{+0.48}$ \\
\hline-20.0 & $0.309 \pm 0.003$ & $0.179 \pm 0.004$ & $0.98 \pm 0.01$ & $2.72_{-0.0 .54}^{+0.4}$ & $0.297 \pm 0.003$ & $0.188 \pm 0.004$ & $0.98 \pm 0.01$ & $2.72_{-0.41}^{+0.58}$ \\
\hline-10.0 & $0.295 \pm 0.003$ & $0.178 \pm 0.004$ & $0.98 \pm 0.01$ & $2.69_{-0.38}^{+0.53}$ & $0.282 \pm 0.003$ & $0.187 \pm 0.004$ & $0.98 \pm 0.01$ & $2.71_{-0.4}^{+0.41}$ \\
\hline 0.0 & $0.282 \pm 0.003$ & $0.177 \pm 0.004$ & $0.98 \pm 0.01$ & $2.68_{-0.38}^{+0.38}$ & $0.268 \pm 0.003$ & $0.187 \pm 0.004$ & $0.98 \pm 0.01$ & $2.73_{-0.42}^{+0.48}$ \\
\hline 10.0 & $0.268 \pm 0.003$ & $0.177 \pm 0.004$ & $0.98 \pm 0.01$ & $\begin{array}{l}-0.38 \\
2.71_{-0.39}^{+0.53}\end{array}$ & $0.254 \pm 0.003$ & $0.185 \pm 0.004$ & $0.98 \pm 0.01$ & $\begin{array}{r}-0.42 \\
2.7_{-0.41}^{+0.58}\end{array}$ \\
\hline 20.0 & $0.255 \pm 0.003$ & $0.176 \pm 0.004$ & $0.98 \pm 0.01$ & $2.7_{-0.37}^{+0.59}$ & $0.24 \pm 0.003$ & $0.184 \pm 0.004$ & $0.98 \pm 0.01$ & $2.71_{-0.4}^{+0.56}$ \\
\hline 30.0 & $0.241 \pm 0.003$ & $0.175 \pm 0.004$ & $0.98 \pm 0.01$ & $2.68_{-0.36}^{+0.51}$ & $0.226 \pm 0.003$ & $0.183 \pm 0.004$ & $0.98 \pm 0.01$ & $2.7_{-0.4}^{+0.55}$ \\
\hline 40.0 & $0.228 \pm 0.003$ & $0.174 \pm 0.004$ & $0.98 \pm 0.01$ & $2.67_{-0.36}^{+0.48}$ & $0.213 \pm 0.003$ & $0.182 \pm 0.004$ & $0.98 \pm 0.01$ & $2.7_{-0.4}^{-0.52}$ \\
\hline 50.0 & $0.215 \pm 0.003$ & $0.173 \pm 0.004$ & $0.98 \pm 0.01$ & $2.68_{-0.36}^{+0.30}$ & $0.199 \pm 0.003$ & $0.181 \pm 0.004$ & $0.98 \pm 0.01$ & $2.69_{-0.38}^{+0.53}$ \\
\hline
\end{tabular}

Table 3. Same as Table 1, except for the Gaia $G$ band, for 5576 stars from the Y18 sample.

as having lower values for $T_{\text {eff }}$ in the APOKASC-2 catalogue itself. However, the fact that APOKASC-2 stars represent a lower-temperature population only accounts for a shift in a measured median absolute magnitude of $\sim 0.028 \mathrm{mag}$ in $K$ and $\sim 0.12 \mathrm{mag}$ in $G$. The use of APOKASC-2 temperatures for the subset shifts the absolute magnitudes even fainter, by another $\sim 0.028$ mag and $\sim 0.07 \mathrm{mag}$ in $K$ and $G$, respectively. At the precision afforded to us by hierarchical models, these shifts caused by the choice of temperatures become statistically significant.

Due to the nature of the $K$ band minimizing the effects of metallicity on the RC spread, there is an extensive literature on the value of $\mu_{\mathrm{RC}}$ in $K$. It was found by Alves (2000) to be $-1.62 \pm 0.03$ (with a consistent measurement 


\begin{tabular}{|c|c|c|c|c|c|c|c|c|}
\hline \multirow[b]{2}{*}{$\Delta T_{\text {eff }}(K)$} & \multicolumn{4}{|c|}{ No Correction } & \multicolumn{4}{|c|}{ Clump Corrected } \\
\hline & $\mu_{\mathrm{RC}}(\mathrm{mag})$ & $\sigma_{\mathrm{RC}}(\mathrm{mag})$ & $Q$ & $\sigma_{\mathrm{o}}\left(\sigma_{\mathrm{RC}}\right)$ & $\mu_{\mathrm{RC}}(\mathrm{mag})$ & $\sigma_{\mathrm{RC}}(\mathrm{mag})$ & $Q$ & $\sigma_{\mathrm{o}}\left(\sigma_{\mathrm{RC}}\right)$ \\
\hline-50.0 & $0.53 \pm 0.004$ & $0.118 \pm 0.006$ & $0.96_{-0.03}^{+0.02}$ & $3.19_{-0.46}^{+0.65}$ & $0.526 \pm 0.004$ & $0.128 \pm 0.005$ & $0.97_{-0.02}^{+0.01}$ & $3.29_{-0.55}^{+0.81}$ \\
\hline-40.0 & $0.516 \pm 0.004$ & $0.117 \pm 0.005$ & $0.96_{-0.03}^{+0.03}$ & $3.18_{-0.46}^{+0.66}$ & $0.51 \pm 0.004$ & $0.127 \pm 0.005$ & $0.97_{-0.02}^{+0.01}$ & $3.31_{-0.55}^{+0.85}$ \\
\hline-30.0 & $0.501 \pm 0.004$ & $0.116 \pm 0.006$ & $0.96_{-0.03}^{+0.02}$ & $3.19_{-0.45}^{+0.62}$ & $0.495 \pm 0.004$ & $0.127 \pm 0.005$ & $0.97_{-0.02}^{+0.01}$ & $3.29_{-0.54}^{+0.76}$ \\
\hline-20.0 & $0.486 \pm 0.004$ & $0.116 \pm 0.006$ & $0.96_{-0.03}^{+0.03}$ & $3.19_{-0.47}^{+0.65}$ & $0.479 \pm 0.004$ & $0.126 \pm 0.005$ & $0.97_{-0.02}^{+0.02}$ & $3.28_{-0.54}^{+0.34}$ \\
\hline-10.0 & $0.472 \pm 0.004$ & $0.115 \pm 0.006$ & $0.96_{-0.03}^{+0.03}$ & $3.19_{-0.45}^{+0.63}$ & $0.464 \pm 0.004$ & $0.126 \pm 0.005$ & $0.97_{-0.02}^{+0.01}$ & $3.29_{-0.55}^{+0.74}$ \\
\hline 0.0 & $0.457 \pm 0.004$ & $0.114 \pm 0.006$ & $0.96_{-0.03}^{+0.02}$ & $3.2_{-0.45}^{+0.64}$ & $0.449 \pm 0.004$ & $0.125 \pm 0.005$ & $0.97_{-0.02}^{+0.01}$ & $3.27_{-0.53}^{+0.79}$ \\
\hline 10.0 & $0.443 \pm 0.004$ & $0.113 \pm 0.006$ & $0.95_{-0.03}^{+0.02}$ & $3.17_{-0.44}^{+0.63}$ & $0.434 \pm 0.004$ & $0.124 \pm 0.005$ & $0.97_{-0.02}^{+0.01}$ & $3.25_{-0.52}^{+0.8}$ \\
\hline 20.0 & $0.429 \pm 0.004$ & $0.113 \pm 0.006$ & $0.96_{-0.03}^{+0.02}$ & $3.21_{-0.44}^{+0.62}$ & $0.419 \pm 0.004$ & $0.124 \pm 0.005$ & $0.97_{-0.02}^{+0.01}$ & $3.25_{-0.53}^{+0.73}$ \\
\hline 30.0 & $0.414 \pm 0.004$ & $0.112 \pm 0.006$ & $0.95_{-0.03}^{+0.02}$ & $3.18_{-0.43}^{+0.61}$ & $0.404 \pm 0.004$ & $0.123 \pm 0.005$ & $0.97_{-0.02}^{+0.01}$ & $3.25_{-0.54}^{+0.72}$ \\
\hline 40.0 & $0.4 \pm 0.004$ & $0.112 \pm 0.006$ & $0.95_{-0.03}^{+0.03}$ & $3.19_{-0.44}^{+0.59}$ & $0.389 \pm 0.004$ & $0.122 \pm 0.005$ & $0.97_{-0.02}^{+0.02}$ & $3.24_{-0.5}^{+0.72}$ \\
\hline 50.0 & $0.386 \pm 0.004$ & $0.111 \pm 0.006$ & $0.95_{-0.03}^{+0.02}$ & $3.2_{-0.43}^{+0.54}$ & $0.375 \pm 0.004$ & $0.122 \pm 0.006$ & $\begin{array}{l}0.97_{-0.02}^{+0.01} \\
x_{-0.0}\end{array}$ & $3.25_{-0.51}^{+0.71}$ \\
\hline
\end{tabular}

Table 4. Same as Table 1, except for the Gaia $G$ band, for a subsample of stars from the APOKASC-2 (Pinsonneault et al. 2018) sample.

\begin{tabular}{rrrrrrr}
\hline$\Delta T_{\text {eff }}(K)$ & \multicolumn{1}{c}{$\mu_{\mathrm{RC}}(\mathrm{mag})$} & \multicolumn{1}{c}{$\sigma_{\mathrm{RC}}(\mathrm{mag})$} & $Q$ & \multicolumn{1}{c}{$\sigma_{\mathrm{o}}\left(\sigma_{\mathrm{RC}}\right)$} & $L(\mathrm{pc})$ & \multicolumn{1}{c}{$\varpi_{\mathrm{zp}}(\mu \mathrm{as})$} \\
\hline $\mathbf{- 5 0 . 0}$ & $-1.71 \pm 0.002$ & $0.041 \pm 0.003$ & $0.58_{-0.05}^{+0.05}$ & $5.49_{-0.47}^{+0.52}$ & $908.63_{-15.89}^{+16.55}$ & $-24.09_{-12}^{+12.84}$ \\
$\mathbf{0 . 0}$ & $-1.737 \pm 0.002$ & $0.04 \pm 0.003$ & $0.55_{-0.03}^{+0.05}$ & $5.61_{-0.47}^{+0.5}$ & $920.12_{-16.61}^{+17.18}$ & $-19.5_{-12}^{+12.4}$ \\
$\mathbf{5 0 . 0}$ & $-1.764 \pm 0.002$ & $0.041 \pm 0.004$ & $0.53_{-0.02}^{+0.04}$ & $5.5_{-0.48}^{+0.5}$ & $930.95_{-16.83}^{+18.07}$ & $-14.81_{-12.98}^{+12.57}$ \\
\hline
\end{tabular}

Table 5. Medians of the posterior distributions for hyperparameters of our Gaia model, for the 2MASS $K$ band, for a randomly selected subsample of 1000 stars from the Y18 sample. Uncertainties are taken as the $1 \sigma$ credible intervals, and are listed as a single value for cases where the posterior was approximately Gaussian. Priors were imposed on $\mu_{\mathrm{RC}}$ and $\sigma_{\mathrm{RC}}$ corresponding to the results for these values using seismic Clump Corrected data in Table 1, for the temperature shifts shown in the $\Delta T_{\text {eff }}$ column. $L$ is the length scale of the exponentially decaying space density prior on distance (Bailer-Jones et al. 2018), and $\varpi_{\mathrm{zp}}$ is the parallax zero-point offset. All other symbols are the same as for Table 1 .

\begin{tabular}{rcrrrrr}
\hline$\Delta T_{\text {eff }}(K)$ & \multicolumn{1}{c}{$\mu_{\mathrm{RC}}(\mathrm{mag})$} & \multicolumn{1}{c}{$\sigma_{\mathrm{RC}}(\mathrm{mag})$} & \multicolumn{1}{c}{$Q$} & \multicolumn{1}{c}{$\sigma_{\mathrm{o}}\left(\sigma_{\mathrm{RC}}\right)$} & \multicolumn{1}{c}{$L(\mathrm{pc})$} & \multicolumn{1}{c}{$\varpi_{\mathrm{zp}}(\mu \mathrm{as})$} \\
\hline $\mathbf{- 5 0 . 0}$ & $-1.661 \pm 0.003$ & $0.04 \pm 0.003$ & $0.6 \pm 0.05$ & $5.76_{-0.5}^{+0.55}$ & $888_{-15.78}^{+16.38}$ & $-33.53_{-12.97}^{+12.93}$ \\
$\mathbf{0 . 0}$ & $-1.689 \pm 0.003$ & $0.04 \pm 0.004$ & $0.59 \pm 0.05$ & $5.66_{-0.51}^{+0.53}$ & $899.36_{-16.04}^{+166.72}$ & $-28.33_{-12.92}^{+12.96}$ \\
$\mathbf{5 0 . 0}$ & $-1.715 \pm 0.003$ & $0.041 \pm 0.004$ & $0.57 \pm 0.05$ & $5.51_{-0.49}^{+0.55}$ & $910.68_{-16.4}^{+168.83}$ & $-23.47_{-13.13}^{+13.25}$ \\
\hline
\end{tabular}

Table 6. Same as Table 5, except for priors imposed on $\mu_{\mathrm{RC}}$ and $\sigma_{\mathrm{RC}}$ corresponding to the results for these values using seismic Clump Corrected data in Table 2, for the temperature shifts shown in the $\Delta T_{\text {eff }}$ column.

by Udalski 2000), but later placed at $-1.54 \pm 0.04$ by Groenewegen (2008). A recent review by Girardi (2016) found a median literature value of $-1.59 \pm 0.04 \mathrm{mag}$, which was applied by Davies et al. (2017) to calibrate TGAS parallaxes. New work by Chen et al. (2017) has used RC stars similarly identified using asteroseismology to find $-1.626 \pm 0.057 \mathrm{mag}$, and the precursor to our hierarchical Bayesian approach, H17, used TGAS parallaxes to find $-1.61 \pm 0.01 \mathrm{mag}$. Using the same method, H17 reported an absolute magnitude of $0.44 \pm 0.01 \mathrm{mag}$ in the Gaia $G$ band.

Our RC magnitudes for both the $K$ and Gaia $G$ bands are much closer to those reported in literature when we used APOKASC-2 stars and temperatures alone. For the $K$ band, we found values within $1 \sigma$ of Chen et al. (2017) for $\Delta T_{\text {eff }} \leq 20 \mathrm{~K}$ when using corrections to the scaling relations, although our results are otherwise incompatible with the literature for $K$. In the $G$ band, however, we found values for $\mu_{\mathrm{RC}}$ compatible with $\mathrm{H} 17$ when using APOKASC-2 stars for $\Delta T_{\text {eff }}$ of 0 or $+10 \mathrm{~K}$ both with and without corrections to the scaling relations. The disagreement found only in the $K$ band could be due to our choice of bolometric corrections or corrections to the scaling relations, or due to H17's choice of extinction coefficient, which is twice as large as the coefficient we use in our Gaia models, and would bias the absolute magnitudes of their stars towards brighter values. Alternatively, it could be due to H17 not accounting for known spatial correlations in parallax (Lindegren et al. 2016; Zinn et al. 2017) or possible parallax zero-point offsets (Brown 2018).

In Tables $9 \& 10$, we attempt to recreate the H17 work, albeit including parallax covariances, and find values for $\mu_{\mathrm{RC}}$ that are compatible with a temperature offset of $\Delta T_{\text {eff }}<-50 \mathrm{~K}$ for both photometric bands. Finally, allowing $\varpi_{\mathrm{zp}}$ to vary as a free parameter with loose prior constraints finds $\mu_{\mathrm{RC}}=-1.634 \pm 0.018 \mathrm{mag}$ in the $K$ band and $0.546 \pm 0.016 \mathrm{mag}$ in the $G$ band. These values imply that a shift to the temperature scales of $-50 \mathrm{~K}$ or more is appropriate when using temperatures for seismology of the Red Clump.

\subsection{Spread of the Red Clump}

In principle, the spread of the RC, like its luminosity, is a property of a RC population and depends on the mass and metallicity of the sample (Girardi 2016; Salaris \& Girardi 2002). Our hierarchical approach allows us to study 


\begin{tabular}{rcccrrr}
\hline$\Delta T_{\text {eff }}(K)$ & $\mu_{\mathrm{RC}}(\mathrm{mag})$ & \multicolumn{1}{c}{$\sigma_{\mathrm{RC}}(\mathrm{mag})$} & $Q$ & \multicolumn{1}{c}{$\sigma_{\mathrm{o}}\left(\sigma_{\mathrm{RC}}\right)$} & \multicolumn{1}{c}{$L(\mathrm{pc})$} & \multicolumn{1}{c}{$\varpi_{\mathrm{zp}}(\mu \mathrm{as})$} \\
\hline $\mathbf{- 5 0 . 0}$ & $0.346 \pm 0.003$ & $0.19 \pm 0.003$ & $0.97_{-0.02}^{+0.01}$ & $3.1_{-0.7}^{+0.79}$ & $948.41_{-17.96}^{+18.15}$ & \multicolumn{1}{c}{$-9.96_{-13}^{+13.1}$} \\
$\mathbf{0 . 0}$ & $0.277 \pm 0.003$ & $0.188 \pm 0.004$ & $0.95_{-0.05}^{+0.02}$ & $2.64_{-0.63}^{+0.77}$ & $978.9_{-17.46}^{+18.95}$ & $1.14_{-12}^{+12.81}$ \\
$\mathbf{5 0 . 0}$ & $0.209 \pm 0.003$ & $0.184 \pm 0.004$ & $0.74_{-0.13}^{+0.12}$ & $1.71_{-0.2}^{+0.36}$ & $1008.77_{-18.48}^{+18.85}$ & $10.76_{-13.21}^{+13.13}$ \\
\hline
\end{tabular}

Table 7. Same as Table 5, except for the Gaia $G$ band, with priors imposed on $\mu_{\mathrm{RC}}$ and $\sigma_{\mathrm{RC}}$ corresponding to the results for these values using seismic Clump Corrected data in Table 3, for the temperature shifts shown in the $\Delta T_{\text {eff }}$ column.

\begin{tabular}{ccccccc}
\hline$\Delta T_{\text {eff }}(K)$ & $\mu_{\mathrm{RC}}(\mathrm{mag})$ & $\sigma_{\mathrm{RC}}(\mathrm{mag})$ & $Q$ & $\sigma_{\mathrm{o}}\left(\sigma_{\mathrm{RC}}\right)$ & $L(\mathrm{pc})$ & $\varpi_{\mathrm{zp}}(\mu \mathrm{as})$ \\
\hline $\mathbf{- 5 0 . 0}$ & $0.527 \pm 0.004$ & $0.13 \pm 0.005$ & $0.82_{-0.07}^{+0.05}$ & $2.53_{-0.3}^{+0.36}$ & $874.12_{-16.1}^{+16.56}$ & $-39.02_{-13.16}^{+12.98}$ \\
$\mathbf{0 . 0}$ & $0.455 \pm 0.004$ & $0.129 \pm 0.005$ & $0.79_{-0.08}^{+0.07}$ & $2.42_{-0.29}^{+0.36}$ & $903.23_{-16.68}^{+16.8}$ & $-26.84_{-12.97}^{+13.1}$ \\
$\mathbf{5 0 . 0}$ & $0.385 \pm 0.004$ & $0.127 \pm 0.005$ & $0.68_{-0.1}^{+0.09}$ & $2.22_{-0.22}^{+0.27}$ & $931.92_{-17}^{+17.53}$ & $-14.94_{-13.04}^{+12.58}$ \\
\hline
\end{tabular}

Table 8. Same as Table 5, except for the Gaia $G$ band, with priors imposed on $\mu_{\mathrm{RC}}$ and $\sigma_{\mathrm{RC}}$ corresponding to the results for these values using seismic Clump Corrected data in Table 4 , for the temperature shifts shown in the $\Delta T_{\text {eff }}$ column.

\begin{tabular}{|c|c|c|c|c|c|c|c|}
\hline Source & $\varpi_{\mathrm{zp}}$ prior $(\mu \mathrm{as})$ & $\mu_{\mathrm{RC}}(\mathrm{mag})$ & $\sigma_{\mathrm{RC}}(\mathrm{mag})$ & $Q$ & $\sigma_{\mathrm{o}}\left(\sigma_{\mathrm{RC}}\right)$ & $L(\mathrm{pc})$ & $\varpi_{\mathrm{zp}}(\mu \mathrm{as})$ \\
\hline Lindegren +18 & $\mathcal{N}(-29.0,1.0)$ & $-1.638 \pm 0.017$ & $0.075_{-0.015}^{+0.016}$ & $0.78_{-0.11}^{+0.09}$ & $3.28_{-0.56}^{+0.64}$ & $888.56_{-24.36}^{+25.41}$ & $-29.07_{-0.99}^{+1}$ \\
\hline Zinn +18 & $\mathcal{N}(-52.8,3.4)$ & $-1.631 \pm 0.017$ & $0.074_{-0.015}^{+0.015}$ & $0.77_{-0.1}^{+0.09}$ & $3.3_{-0.57}^{+0.65}$ & $885.76_{-23.73}^{+24.40}$ & $-51.92_{-3.21}^{+3.21}$ \\
\hline Riess+ 18 & $\mathcal{N}(-46.0,13.0)$ & $-1.634 \pm 0.017$ & $0.076_{-0.015}^{+0.017}$ & $0.78_{-0.11}^{+0.09}$ & $3.26_{-0}^{+0.64}$ & $886.59_{-24.12}^{+\overline{2} 5}$ & $-42.22_{-9.16}^{+9.33}$ \\
\hline Sahlholdt \& Silva Aguirre18 & $\mathcal{N}(-35.0,16.0)$ & $-1.634 \pm 0.017$ & $0.073_{-0.015}^{+0.015}$ & $0.77_{-0.11}^{+0.09}$ & $3.33_{-0.58}^{+0.69}$ & $887.37_{-23.89}^{+24.06}$ & $-37_{-10.42}^{+10.17}$ \\
\hline Stassun \& Torres 18 & $\mathcal{N}(-82.0,33.0)$ & $-1.632 \pm 0.017$ & $0.072_{-0.016}^{+0.017}$ & $0.76_{-0.11}^{+0.09}$ & $3.36_{-0.59}^{+0.60}$ & $885.77_{-23.01}^{+24.39}$ & $-44.55_{-12.59}^{+12.62}$ \\
\hline Hawkins+ 17 & $\mathcal{N}(0.0,1.0)$ & $-1.648 \pm 0.018$ & $0.075_{-0.015}^{+0.010}$ & $0.78_{-0.11}^{+0.09}$ & $3.31_{-0.57}^{+0.64}$ & $893.39_{-24}^{+24.6}$ & $-0.22_{-1.01}^{+0.99}$ \\
\hline Uninformed & $\mathcal{N}(0.0,1000.0)$ & $-1.634 \pm 0.018$ & $0.074_{-0.015}^{+0.017}$ & $0.77_{-0.11}^{+0.09}$ & $3.3_{-0.58}^{+0.64}$ & $887.27_{-23.82}^{+24.12}$ & $-38.38_{-13.54}^{+13.83}$ \\
\hline
\end{tabular}

Table 9. Medians on the posterior distributions for hyperparameters on our Gaia model, for the 2MASS $K$ band, for a randomly selected subsample of 1000 stars from the Y18 sample. Uncertainties are taken as the $1 \sigma$ credible intervals, and are listed as single values for cases where the posterior was approximately Gaussian. Highly informative priors, shown in the ' $\varpi_{\mathrm{zp}}$ prior' column, were imposed on $\varpi_{\mathrm{zp}}$ corresponding to estimates for this parameter from the literature, listed in bold print in the Source column. Additionally, we apply a custom prior to place $\varpi_{\mathrm{zp}}$ near zero in order to recreate conditions similar to the H17 work, and an extremely broad prior on $\varpi_{\mathrm{zp}}$ in order to find a value given no strong constraints on neither $\varpi_{\mathrm{zp}}, \mu_{\mathrm{RC}}$ or $\sigma_{\mathrm{RC}}$. $\mathcal{N}(\mu, \sigma)$ indicates a normal distribution with mean $\mu$ and standard deviation $\sigma$.

the 'true' spread of the RC, by evaluating the uncertainties on individual measures of absolute magnitude.

As seen for the $K$ band in Tables $1 \& 2$, the spread of the $\mathrm{RC}$ is consistent within $1 \sigma$ for all perturbations of temperature, corrections to the scaling relations, and between both the Yu et al. (2018) and APOKASC-2 temperatures. This indicates that $\sigma_{\mathrm{RC}}$ is only weakly dependent on the choice of temperature scale, and that any effects of the APOKASC-2 sample only representing a small subset in metallicity are minimal for the $K$ band.

The spread of the RC due to mass and metallicity is minimised in the 2MASS $K$ band (Salaris \& Girardi 2002), which would lead us to expect a broader spread of the RC in the Gaia $G$ band. We see this effect in Tables $3 \& 4$, where the reported spreads are $\sim 4$ to 6 times larger in magnitude. Surprisingly, we do not see the same consistency for the values of $\sigma_{\mathrm{RC}}$ for the $G$ band, but instead find that the inferred value of $\sigma_{\mathrm{RC}}$ varies inversely with temperature beyond $1 \sigma$ from $-50 \mathrm{~K}$ to $50 \mathrm{~K}$. This trend of $\sigma_{\mathrm{RC}}$ with $\Delta T_{\text {eff }}$ is likely to be an effect of the bolometric correction, as we do not see a compatible trend in $K$. It should also be noted that we would expect extinction to play a larger role in the $G$ band, possibly contributing to this effect.

For the Gaia $G$ band we also see that the value for $\sigma_{\mathrm{RC}}$ is lower for the APOKASC-2 sample than for the full Y18 sample. This reduction is liklely because the APOKASC2 sample draws temperatures from a uniform spectroscopy source (and thus temperature scale) whereas the Y18 temperatures come from a variety of sources, broadening the distribution of RC stars.

The similar hierarchical approach taken by $\mathrm{H} 17$ found a spread of $0.17 \pm 0.02 \mathrm{mag}$ in $K$ and $0.20 \pm 0.02 \mathrm{mag}$ in $G$ using TGAS parallaxes. The agreement within $1 \sigma$ for the $G$ band for the Y18 sample agrees with the inferred APOKASC2 spread being an underestimate. The estimates found in our work for $\sigma_{\mathrm{RC}}$ in $K$ are an order of magnitude lower. This is probably due to our sample size (increased from H17 by a factor of 5) and asteroseismology providing more precise measurements for these stars than TGAS (Davies et al. 2017), allowing the hierarchical method to more closely constrain the true underlying spread.

Tables $9 \& 10$ show the results of our attempt to recreate the H17 work, accounting for parallax covariances and including a parallax zero-point offset. Using Gaia parallaxes, we found a $\sigma_{\mathrm{RC}}$ in $K$ that is larger than our value from seismology. The results presented in Tables $5 \& 6$, where the the seismic $\sigma_{\mathrm{RC}}$ in $K$ has been applied as a prior on the Gaia model, show an inlier fraction $Q$ that is lower than we would expect for this sample. This implies that Gaia DR2 is underestimating the uncertainties for stars considered 'outliers', and not including them in the inlier population.

For the $G$ band, we found a value for $\sigma_{\mathrm{RC}}$ in agreement with our seismic value using APOKASC-2 temperatures. In this instance, as opposed to the results shown in Table 8 


\begin{tabular}{|c|c|c|c|c|c|c|c|}
\hline Source & $\varpi_{\mathrm{zp}}$ prior $(\mu \mathrm{as})$ & $\mu_{\mathrm{RC}}(\mathrm{mag})$ & $\sigma_{\mathrm{RC}}(\mathrm{mag})$ & $Q$ & $\sigma_{\mathrm{o}}\left(\sigma_{\mathrm{RC}}\right)$ & $L(\mathrm{pc})$ & $\varpi_{\mathrm{zp}}(\mu \mathrm{as})$ \\
\hline Lindegren +18 & $\mathcal{N}(-29.0,1.0)$ & $0.542 \pm 0.016$ & $0.138_{-0.018}^{+0.014}$ & $0.86_{-0.12}^{+0.07}$ & $2.61_{-0.34}^{+0.48}$ & $868.2_{-17.09}^{+17.41}$ & $-29.06_{-1.01}^{+0.98}$ \\
\hline Zinn +18 & $\mathcal{N}(-52.8,3.4)$ & $0.548 \pm 0.016$ & $0.139_{-0.018}^{+0.018}$ & $0.86_{-0.12}^{+0.07}$ & $2.62_{-0.35}^{+0.49}$ & $865.44_{-17.15}^{+16.95}$ & $-52.18_{-3.31}^{+3.91}$ \\
\hline Riess +18 & $\mathcal{N}(-46.0,13.0)$ & $0.545 \pm 0.016$ & $0.14_{-0.017}^{+0.018}$ & $0.87_{-0.11}^{+0.07}$ & $2.62_{-0.34}^{+0.48}$ & $867.13_{-17.25}^{+11.23}$ & $-44.23_{-9.32}^{+9: 06}$ \\
\hline Sahlholdt \& Silva Aguirre 18 & $\mathcal{N}(-35.0,16.0)$ & $0.545 \pm 0.016$ & $0.136_{-0.021}^{+0.0115}$ & $0.85_{-0.14}^{+0.08}$ & $2.62_{-0.34}^{+0.44}$ & $867.15_{-17.05}^{+17.35}$ & $-39.29_{-10.27}^{+9.862}$ \\
\hline Stassun \& Torres 18 & $\mathcal{N}(-82.0,33.0)$ & $0.546 \pm 0.017$ & $0.138_{-0.018}^{+0.011}$ & $0.86_{-0.12}^{+0.07}$ & $2.61_{-0.33}^{+0.46}$ & $866.11_{-17.02}^{+17.75}$ & $-47.86_{-12.51}^{+12.18}$ \\
\hline Hawkins +17 & $\mathcal{N}(0.0,1.0)$ & $0.534 \pm 0.015$ & $0.14_{-0.018}^{+0.018}$ & $0.87_{-0.12}^{-0.12}$ & $2.64_{-0.35}^{+0.33}$ & $872.01_{-17.38}^{+17.02}$ & $-0.23_{-1.01}^{+1.51}$ \\
\hline Uninformed & $\mathcal{N}(0.0,1000.0)$ & $0.546 \pm 0.016$ & $0.139_{-0.019}^{+0.018}$ & $0.87_{-0.13}^{+0.07}$ & $2.62_{-0.34}^{+0.49}$ & $866.26_{-16.86}^{+17: 53}$ & $-42.66_{-13.14}^{+13.48}$ \\
\hline
\end{tabular}

Table 10. Same as Table 9, except for the Gaia $G$ band.

at similar $\sigma_{\mathrm{RC}}$, we find an inlier fraction $Q$ in the expected range. This is probably due to the simultaneous inference of a more appropriate value for $\mu_{\mathrm{RC}}$, which is closer to values established in literature (H17). For this reason, the spreads reported in Tables $4 \& 10$ are our best estimates for the 'true' spread of the RC in the $G$ band.

With our measurement of $\sigma_{\mathrm{RC}}=0.03 \mathrm{mag}$ in the $K$ band, we can use standard error propagation through equation 20 (setting extinction to zero) to find that this spread yields a precision in distance of $\sim 1 \%$ for our sample, subject to mass and metallicity. This is a factor of 5 improvement from the precision reported by H17. When using $\sigma_{\mathrm{RC}}=0.14$ mag for the $G$ band we find a distance precision of $\sim 6 \%$, in line with the findings by $\mathrm{H} 17$.

\subsection{The Gaia parallax zero-point offset}

The Gaia DR2 parallax zero-point offset, while small, can still have an effect on results, and is widely applied in studies using DR2 (Luri et al. 2018; Bailer-Jones et al. 2018), with potentially far-reaching consequences (Shanks et al. 2019). The offset has been estimated through calibration with eclipsing binaries (Stassun \& Torres 2018), Cepheids (Riess et al. 2018), asteroseismology (Zinn et al. 2018; Sahlholdt \& Silva Aguirre 2018), kinematics (Schönrich et al. 2019) and quasars (Lindegren et al. 2018).

In Tables 5, 6, 7 and 8 we present our inferred model parameters given our values for $\mu_{\mathrm{RC}}$ and $\sigma_{\mathrm{RC}}$ found through asteroseismology at different temperature shifts $\Delta T_{\text {eff }}$, effectively 'calibrating' Gaia DR2 to see what offset recovers a given set of RC parameters.

Figure 7 shows the posterior distributions for $\varpi_{\text {zp }}$ given our seismic priors from different temperature shifts, where there is a clear trend of $\varpi_{\mathrm{zp}}$ with seismic $\mu_{\mathrm{RC}}$, and thus with temperature. This trend was also found in recent results by Khan et al. (2019), where a comparison of Gaia parallaxes and seismic distances obtained through the seismic scaling relations found that a temperature shift of $100 \mathrm{~K}$ caused a shift in $\varpi_{\mathrm{zp}}$ of $10-15 \mu$ as for RC stars, although it should be noted that they found this effect largely reduced when using grid modelling techniques (Rodrigues et al. 2017).

It is also apparent in Figure 7 that the uncertainty on $\varpi_{\text {zp }}$ is significant, and consistent for all model conditions, due to the parallax covariances presenting a systematic lower limit on parallax uncertainties for this sample. Given a $\mu_{\mathrm{RC}}$ in $K$ closer to literature values, with the run corresponding to APOKASC-2 temperatures using $\Delta T_{\text {eff }}=-50 K$, we found a $\varpi_{\mathrm{zp}}$ within $1 \sigma$ of the uncertainties on all literature values for $\varpi_{\mathrm{zp}}$ in the Kepler field discussed in this work. This is both an encouraging sign of a consistent $\varpi_{\mathrm{zp}}$ in the Kepler field, and further indication that seismology would be improved by reducing the temperature scale. For the Gaia $G$ band, the run closest to the existing literature $\left(\Delta T_{\text {eff }}=0\right)$ is consistent with all values for $\varpi_{\mathrm{zp}}$ besides Stassun \& Torres (2018).

Given a selection of values for $\varpi_{\text {zp }}$ reported in the literature, we applied informative priors on $\varpi_{\mathrm{zp}}$ in our Gaia model, and allowed $\mu_{\mathrm{RC}}$ and $\sigma_{\mathrm{RC}}$ to explore the parameter space freely. The results of this are shown in Tables $9 \& 10$, for the $K$ and $G$ bands respectively. The credible intervals for $\mu_{\mathrm{RC}}$ are shown in Figure 8. For both bands, we found that the choice of $\varpi_{\mathrm{zp}}$ from the literature had no impact beyond $1 \sigma$ on either of the RC properties for any values used. When using a tightly constrained $\varpi_{\text {zp }}$ of zero (in an attempt to recreate H17) we found the largest overall change. It is also interesting to note that for a prior corresponding to the Stassun \& Torres (2018) value, the inferred value for $\varpi_{\mathrm{zp}}$ is reduced to lie closer to those found in other works for the Kepler field.

Finally, running the Gaia model with uninformative priors on both $\varpi_{\text {zp }}$ and the RC parameters produced a parallax zero-point offset of $(-38 \pm 13) \mu$ as in $K$ and $(-42 \pm 13) \mu$ as in $G$ for the Kepler field. These values are consistent with one another and with the existing literature, and also agree with recent results by Khan et al. (2019) for RC stars in APOKASC-2. Given the uncertainties on the inferred values of $\varpi_{\mathrm{zp}}$, we see a fundamental uncertainty limit on Gaia parallaxes of $\sim 13 \mu$ as as a result of spatial covariances in the parallaxes. Encouragingly, this implies that for our RC sample in the Kepler field, the choice of parallax-zero point offset does not dramatically impact the inferred luminosities, given a proper treatment of the spatial parallax covariances. However, this may not generalize to populations more sparsely sampled in space, and in other magnitude ranges, given the known relation between the parallax zero-point offset, $G$ band magnitude and colour (Zinn et al. 2018; Lindegren et al. 2018).

\subsection{Corrections to the seismic scaling relations}

In Section 5.3, we have compared results with and without corrections to the $\Delta v$ seismic scaling relation, $f_{\Delta v}$, derived from Sharma \& Stello (2016). It is known that stellar models do not not accurately reproduce the $\Delta v$ of the Sun (off by about $1 \%$ ), due to the so-called surface effect (ChristensenDalsgaard et al. 1988; White et al. 2011). Corrections to the scaling relation $f_{\Delta v}$ derived without accounting for the surface effect (such as Sharma \& Stello 2016) can produce radii that differ on the order of $\sim 2 \%$ from methods that do 
(such as Rodrigues et al. 2017). As a check, we considered the impact that this may have on our inferred values for the RC magnitude.

To compare the calculated RC populations in the $K$ and Gaia $G$ bands, we obtained radii using $f_{\Delta v}$ obtained through Sharma \& Stello (2016). We then used bolometric corrections for no temperature offset to calculate the absolute magnitudes using both those radii and those same radii reduced by both $1.6 \%$ and $2.4 \%$. We found that a reduction on radius in the range $(2 \pm 0.4) \%$ resulted in a global shift toward brighter bolometric magnitudes by $44_{-8}^{+9}$ mmag.

In Tables $2 \& 4$ we report the absolute magnitude of the $\mathrm{RC}$ (for no temperature offset) in the APOKASC-2, $f_{\Delta v^{-}}$ corrected, sample of $-1.69 \mathrm{mag}$ in $K$ and $0.45 \mathrm{mag}$ in $G$. A shift of 0.04 mag applied to both bands is enough to reconcile our seismic results with those obtained through Gaia for both the $K$ and $G$ bands, as well as those from the literature. Note however that this is not the case when applied to the Y18 sample (see Tables $1 \& 3$ ), where this shift applied in both bands would not be enough to reconcile the seismic results for the absolute magnitude of the $\mathrm{RC}$ with any measures both in this work or in the literature.

\subsection{Calibrating Gaia and asteroseismology}

Our initial aim with this work was to calibrate the Gaia parallax zero-point offset, $\varpi_{\text {zp }}$, using asteroseismology. Given the large change in the absolute magnitude of the $\mathrm{RC}, \mu_{\mathrm{RC}}$, with relatively small changes in temperature for our large $\mathrm{RC}$ population, and consequently the shift in inferred $\varpi_{\mathrm{zp}}$ given these values for $\mu_{\mathrm{RC}}$, it proved difficult to definitively calibrate Gaia parallaxes using seismology.

The reverse however, seems more possible. We found that the various parallax offsets reported in the literature, when used as informative priors on our Gaia model, all resulted in similar values for $\mu_{\mathrm{RC}}$ in both the 2MASS $K$ and Gaia $G$ bands (as shown in Tables $9 \& 10$ ), and inferred values for $\varpi_{\text {zp }}$ that lie closer together for those literature values with large uncertainties (Stassun \& Torres 2018; Riess et al. 2018; Sahlholdt \& Silva Aguirre 2018). Imposing a prior for $\varpi_{\text {zp }}$ to lie close to zero showed a departure beyond $1 \sigma$ from the $\mu_{\mathrm{RC}}$ values found otherwise, indicating that $\varpi_{\mathrm{zp}}$ does have a measurable effect on the inferred RC luminosity. Finally, applying no strongly informative priors on the $\mathrm{RC}$ parameters nor $\varpi_{\mathrm{zp}}$ led to inferred values of $\mu_{\mathrm{RC}}$ and $\varpi_{\mathrm{zp}}$ being consistent with values in the literature, albeit with a large uncertainty of $\sim 13 \mu$ as on the parallax zero-point offset, implying a fundamental limit on the uncertainty on this offset given the spatial parallax covariances.

Given that the choice of parallax zero-point offset did not dramatically affect the inferred luminosity of the clump (see Tables $9 \& 10$ and Figure 8 ), we can reasonably use any value of $\varpi_{\mathrm{zp}}$ reported in the literature, including from this work, to attempt a calibration of seismology. Given the results for our runs on Gaia data with RC parameters constrained by seismology (Tables $6 \& 8$ ), we expect that $\mu_{\mathrm{RC}}=-1.634 \mathrm{mag}$ in $K$ and in $0.546 \mathrm{mag}$ in $G$ would be roughly consistent with a temperature offset $\Delta T_{\text {eff }}$ between $\sim-100 K$ and $\sim-70 K$ for temperatures in the APOKASC-2 catalogue ((which, as has been noted, are already lower than those reported by Mathur et al. 2017, for the same stars). An offset of this size would fall within known systematic un- certainties on temperatures inferred from seismology (Slumstrup et al. 2019). However, it should be noted that this shift in temperature scale is degenerate with the scaling relations underestimating radii by $\sim 2 \%$ compared to our estimates for radius using corrections by Sharma \& Stello (2016), as discussed in section 5.4.

In order to confirm these proposed shifts to temperature, we reran our asteroseismic model on our APOKASC-2 subsample for a range of temperature shifts extended down to $-110 K$ for both the $K$ and Gaia $G$ bands, with RCcorrected scaling relations. We found that when considering the $K$ band, our calibration value for $\mu_{\mathrm{RC}}$ from Gaia corresponds to within $1 \sigma$ with a temperature shift of between -110 and $-70 \mathrm{~K}$. When considering the $G$ band, the Gaia $\mu_{\mathrm{RC}}$ corresponds to within $1 \sigma$ for a shift between -70 and $-50 \mathrm{~K}$. Given that any calibrated correction to the temperature scale should be applied globally to the full APOKASC-2 subsample, we find that a temperature shift of $-70 \mathrm{~K}$ to the temperatures of our RC subsample of APOKASC-2 would produce seismic absolute magnitudes of the clump consistent with those found using Gaia DR2.

We only ran this test for the APOKASC-2 subsample, for which temperatures were all drawn from a uniform spectroscopic source. Since the temperatures for the full Y18 are not, claims about changes to temperature scales for this sample would be inappropriate.

The ability to make this inference reliably rests on our hierarchical treatment, as initially set out by H17, and treatment of the spatial correlations in parallax reported by Lindegren et al. (2018). As we improve our understanding of these correlations, our inferences using this and similar hierarchical models will improve. Similarly, it is known that population effects in age, metallicity and temperature, among others, have an effect on the inferred luminosity of the RC Girardi (2016). Our hierarchical model, can be further improved by accounting for these effects, as well as including parameters that check for consistent colours, as suggested by H17. As these hierarchical models improve in future work, so will our understanding of the RC, and our ability to calibrate asteroseismology.

\section{CONCLUSIONS}

Using two hierarchical models, based on the work by H17, we inferred the spread and position in absolute magnitude of a sample of 5576 Red Clump (RC) stars in the 2MASS $K$ and Gaia $G$ bands. We first did this using absolute magnitudes obtained through a completely distance-independent asteroseismic method, and probed systematics in asteroseismology by varying the temperatures of the sample, applying corrections to the scaling relations, and running our model on a subsample of stars with separate spectroscopic temperatures reported in APOKASC-2 (Pinsonneault et al. 2018). We then applied the results from seismology as strongly informative priors on the position and spread of the clump for our second hierarchical model. We applied this to Gaia DR2 data in order to see how the parallax zero-point varied, taking into account spatial correlations of parallaxes reported by Lindegren et al. (2018). We then applied strongly informative priors on the parallax zero-point in our Gaia model and allowed the $\mathrm{RC}$ parameters to roam more freely, to study 
the impact of published values for the zero-point offset on the RC. Finally, we performed a run of the Gaia model with no strongly informative priors on any parameters.

We leave the reader with the following conclusions:

(i) By applying the $\mathrm{H} 17$ hierarchical model, with improvements to account for spatial correlations of parallaxes and maginalize over the parallax zero-point offset $\left(\varpi_{\mathrm{zp}}\right)$, we find a mean value for $\varpi_{\mathrm{zp}}$ in the Kepler field to be $-41 \pm 10 \mu$ as for our sample, consistent with all existing measures of $\varpi_{\mathrm{zp}}$ in the Kepler field. This offset results in a Red Clump magnitude of $-1.634 \pm 0.018$ in $K$ and $0.546 \pm 0.016$ in $G$ for our sample.

(ii) Applying a hierarchical model to our sample of absolute magnitudes obtained from asteroseismology, we find a spread of the RC in the 2MASS $K$ band of $\sim 0.03$ mag independent of our changes made to the sample, an order of magnitude lower than the value reported previously using Gaia TGAS parallaxes in H17. This extremely small spread highlights the power of seismology and the potential of the $\mathrm{RC}$ in the $K$ band as a standard candle. In the Gaia $G$ band we find a spread of $\sim 0.13$ mag using APOKASC- 2 temperatures, which is consistent with results found using Gaia DR2 parallaxes.

(iii) We find that a small global change in temperature $(\sim 10-20 \mathrm{~K})$ can affect the inferred absolute magnitude of the $\mathrm{RC}$ from seismology by more than $1 \sigma$, and is degenerate with the application of a correction $f_{\Delta v}$ to the seismic scaling relations.

(iv) We find values for the absolute magnitude of the $\mathrm{RC}$ from seismology to agree within $1 \sigma$ with those inferred from Gaia DR2 parallaxes in both the $K$ and $G$ bands, only if a global temperature shift of $\sim-70 \mathrm{~K}$ is applied to our RC subsample of APOKASC-2 stars. This shift is within expected systematic uncertainties on spectroscopic techniques. These differences are also degenerate with a shift in seismic radius of $2 \%$, which is within the uncertainty imposed by choice of corrections to the scaling relations.

(v) A hierarchical Bayesian mixture model for a population of RC stars, as first set out by H17, continues to be an excellent tool for working with Gaia DR2 parallaxes, with the new additions of a parallax zero-point offset as a parameter and spatial correlations between parallaxes. Further additions will undoubtedly improve our inferences on RC stars, and with it, our ability to calibrate asteroseismology and Gaia.

\section{ACKNOWLEDGEMENTS}

The authors would like to thank the anonymous reviewer for their helpful comments, which contributed to the quality of this manuscript. They would also like to thank Benoît Mosser, Marc Pinsonneault, Joel Zinn and James Kuszlewicz for the helpful discussions. OJH, GRD, YPE and WJC acknowledge the support of the UK Science and Technology Facilities Council (STFC). AM acknowledges support from the ERC Consolidator Grant funding scheme (project ASTEROCHRONOMETRY, G.A. n. 772293). AGAB acknowledges financial support from the Netherlands Research School for Astronomy (NOVA). KH is partially supported by a Research Corporation TDA Grant. RAG acknowledges the support from the PLATO CNES grant. This work has made use of data from the European Space Agency (ESA) mission Gaia (https://www.cosmos.esa.int/gaia), processed by the Gaia. Data Processing and Analysis Consortium (DPAC, https://www.cosmos.esa.int/web/gaia/ dpac/consortium). Funding for the DPAC has been provided by national institutions, in particular the institutions participating in the Gaia Multilateral Agreement. This publication makes use of data products from the Two Micron All Sky Survey, which is a joint project of the University of Massachusetts and the Infrared Processing and Analysis Center/California Institute of Technology, funded by the National Aeronautics and Space Administration and the National Science Foundation.

SOFTWARE: This work has made use of the following software not cited in the text: astropy (Astropy Collaboration et al. 2013, 2018), corner (Foreman-Mackey 2016), daft (Foreman-Mackey \& Hogg 2012), PyStan (Stan Development Team 2018), Stan (Carpenter et al. 2017), scikitlearn (Pedregosa et al. 2011), iPython (Pérez \& Granger 2007), Jupyter Notebooks (Kluyver et al. 2016), numpy (Travis E 2006), pandas (McKinney 2010), matplotlib (Hunter 2007) and seaborn (Waskom et al. 2017).

\section{REFERENCES}

Alves D. R., 2000, Astrophys. J., 539, 732

Arenou F., et al., 2018, A\&A, 616, A17

Astraatmadja T. L., Bailer-Jones C. A. L., 2016a, Astrophys. J., 832,137

Astraatmadja T. L., Bailer-Jones C. A. L., 2016b, Astrophys. J., 833,119

Astraatmadja T. L., Bailer-Jones C., 2017, Am. Astron. Soc. Meet. Abstr., p. 134.06

Astropy Collaboration et al., 2013, Astronomy and Astrophysics, 558, A33

Astropy Collaboration et al., 2018, The Astronomical Journal, 156,123

Baglin A., Michel E., Auvergne M., Team C., 2006, Proc. SOHO 18GONG 2006HELAS Spherical Sun, 624, 34

Bailer-Jones C. A. L., 2015, Publ. Astron. Soc. Pac., 127, 994

Bailer-Jones C. a. L., Rybizki J., Fouesneau M., Mantelet G., Andrae R., 2018, Astron. J., 156, 58

Batalha N. M., et al., 2011, Astrophys. J., 729, 27

Beck P. G., De Ridder J., Aerts C., Kallinger T., Hekker S., García R. A., Mosser B., Davies G. R., 2012, Astron. Nachrichten, 333,967

Bedding T. R., et al., 2011, Nature, 471, 608

Belkacem K., Goupil M. J., Dupret M. A., Samadi R., Baudin F., Noels A., Mosser B., 2011, Astron. Astrophys., 530, A142

Belkacem K., Samadi R., Mosser B., Goupil M.-J., Ludwig H.-G., 2013, Prog. Phys. Sun Stars New Era Helio- Asteroseismol., 479,61

Bellinger E. P., Hekker S., Angelou G. C., Stokholm A., Basu S., 2019, A\&A, 622, A130

Borucki W. J., Koch D., Team K. S., 2010, AASDivision Planet. Sci. Meet. Abstr. 42, 42, 47.03

Brogaard K., et al., 2018, Mon. Not. R. Astron. Soc., 476, 3729

Brown A. G. A., 2018, Astrom. Astrophys. Gaia Sky, 330, 13

Brown T. M., Gilliland R. L., Noyes R. W., Ramsey L. W., 1991, Astrophys. J., 368, 599

Cannon R. D., 1970, Mon. Not. R. Astron. Soc., 150, 111

Carpenter B., et al., 2017, J. Stat. Softw., 76, 1

Casagrande L., VandenBerg D. A., 2014, Mon. Not. R. Astron. Soc., 444, 392 
Casagrande L., VandenBerg D. A., 2018a, Mon. Not. R. Astron. Soc., 475, 5023

Casagrande L., VandenBerg D. A., 2018b, Mon. Not. R. Astron. Soc., 479, L102

Casagrande L., et al., 2014, Astrophys. J., 787, 110

Casagrande L., et al., 2016, Mon. Not. R. Astron. Soc., 455, 987

Chaplin W. J., Miglio A., 2013, Annu. Rev. Astron. Astrophys., 51,353

Chaplin W. J., et al., 2010, Astrophys. J., 713, L169

Chaplin W. J., et al., 2011, Science, 332, 213

Chaplin W. J., et al., 2013, Astrophys. J., 766, 101

Chaplin W. J., et al., 2014, Astrophys. J. Suppl. Ser., 210, 1

Chen Y. Q., Casagrande L., Zhao G., Bovy J., Silva Aguirre V., Zhao J. K., Jia Y. P., 2017, Astrophys. J., 840, 77

Christensen-Dalsgaard J., Dappen W., Lebreton Y., 1988, Nature, 336,634

Christensen-Dalsgaard J., et al., 2010, Astrophys. J., 713, L164

Davies G. R., et al., 2015, Mon. Not. R. Astron. Soc., 446, 2959

Davies G. R., et al., 2016, Mon. Not. R. Astron. Soc., 456, 2183

Davies G. R., et al., 2017, Astron. Astrophys., 598, L4

Deheuvels S., et al., 2012, Astrophys. J., 756, 19

Deheuvels S., et al., 2014, Astron. Astrophys., 564, A27

Elsworth Y., Hekker S., Basu S., Davies G. R., 2017, Mon. Not. R. Astron. Soc., 466, 3344

Foreman-Mackey D., 2016, The Journal of Open Source Software, 24

Foreman-Mackey D., Hogg D. W., 2012

Gaia Collaboration et al., 2016, Astron. Astrophys., 595, A1

Gaia Collaboration et al., 2018, A\&A, 616, A1

García R. A., et al., 2011, MNRAS, 414, L6

García R. A., et al., 2014, Astronomy and Astrophysics, 568, A10

Gaulme P., et al., 2016, Astrophys. J., 832, 121

Girardi L., 1999, Mon. Not. R. Astron. Soc., 308, 818

Girardi L., 2016, Annu. Rev. Astron. Astrophys., 54, 95

Green G. M., et al., 2018, Mon. Not. R. Astron. Soc., 478, 651

Groenewegen M. a. T., 2008, Astron. Astrophys., 488, 935

Handberg R., Lund M. N., 2014, Monthly Notices of the Royal Astronomical Society, 445, 2698

Hawkins K., Leistedt B., Bovy J., Hogg D. W., 2017, Mon. Not. R. Astron. Soc., 471, 722

Hekker S., et al., 2011, Mon. Not. R. Astron. Soc., 414, 2594

Hogg D. W., Bovy J., Lang D., 2010, ArXiv E-Prints, p. arXiv:1008.4686

Huber D., et al., 2011, Astrophys. J., 743, 143

Huber D., et al., 2013a, Science, 342, 331

Huber D., et al., 2013b, Astrophys. J., 767, 127

Huber D., et al., 2014, Astrophys. J. Suppl. Ser., 211, 2

Huber D., et al., 2017, Astrophys. J., 844, 102

Hunter J. D., 2007, Computing In Science \& Engineering, 9, 90

Jofré E., Petrucci R., Saffe C., Saker L., de la Villarmois E. A., Chavero C., Gómez M., Mauas P. J. D., 2015, Astronomy and Astrophysics, 574, A50

Khan S., Hall O. J., Miglio A., Davies G. R., Mosser B., Girardi L., Montalbán J., 2018, Astrophys. J., 859, 156

Khan S., et al., 2019, ArXiv E-Prints, p. arXiv:1904.05676

Kjeldsen H., Bedding T. R., 1995, Astron. Astrophys., 293, 87

Kluyver T., et al., 2016, IOS Press, pp $87-90$

Lindegren L., et al., 2016, Astron. Astrophys., 595, A4

Lindegren L., et al., 2018, A\&A, 616, A2

Lund M. N., et al., 2017, Astrophys. J., 835, 172

Luri X., et al., 2018, A\&A, 616, A9

Maíz Apellániz J., Weiler M., 2018, Astron. Astrophys., 619, A180

Mathur S., García R. A., Huber D., Regulo C., Stello D., Beck P. G., Houmani K., Salabert D., 2016, Astrophys. J., 827, 50

Mathur S., et al., 2017, Astrophys. J. Suppl. Ser., 229, 30

McKinney W., 2010, pp $51-56$

Metcalfe T. S., Mathur S., Doğan G., Woitaszek M., 2012, Prog. SolarStellar Phys. Helio- Asteroseismol., 462, 213
Metcalfe T. S., et al., 2014, Astrophys. J. Suppl. Ser., 214, 27

Miglio A., et al., 2009, Astron. Astrophys., 503, L21

Miglio A., et al., 2012, Mon. Not. R. Astron. Soc., 419, 2077

Miglio A., et al., 2013, Mon. Not. R. Astron. Soc., 429, 423

Mosser B., et al., 2012a, Astron. Astrophys., 540, A143

Mosser B., et al., 2012b, Astron. Astrophys., 548, A10

Mosser B., Vrard M., Belkacem K., Deheuvels S., Goupil M. J., 2015, Astron. Astrophys., 584, A50

Paxton B., Bildsten L., Dotter A., Herwig F., Lesaffre P., Timmes F., 2011, Astrophys. J. Suppl. Ser., 192, 3

Paxton B., et al., 2013, Astrophys. J. Suppl. Ser., 208, 4

Paxton B., et al., 2015, Astrophys. J. Suppl. Ser., 220, 15

Pedregosa F., et al., 2011, Journal of Machine Learning Research, 12,2825

Pérez F., Granger B. E., 2007, Computing in Science and Engineering, 9,21

Pinsonneault M. H., et al., 2014, Astrophys. J. Suppl. Ser., 215, 19

Pinsonneault M. H., et al., 2018, The Astrophysical Journal Supplement Series, 239, 32

Riess A. G., et al., 2018, Astrophys. J., 861, 126

Rodrigues T. S., et al., 2017, Mon. Not. R. Astron. Soc., 467, 1433

Sahlholdt C. L., Silva Aguirre V., 2018, MNRAS, 481, L125

Salaris M., Girardi L., 2002, Mon. Not. R. Astron. Soc., 337, 332

Schönrich R., McMillan P., Eyer L., 2019, arXiv e-prints, p. arXiv:1902.02355

Shanks T., Hogarth L. M., Metcalfe N., 2019, MNRAS, 484, L64

Sharma S., Stello D., 2016, Astrophys. Source Code Libr., p. ascl:1603.009

Sharma S., Stello D., Bland-Hawthorn J., Huber D., Bedding T. R., 2016, Astrophys. J., 822, 15

Silva Aguirre V., et al., 2013, Astrophys. J., 769, 141

Silva Aguirre V., et al., 2015, Mon. Not. R. Astron. Soc., 452, 2127

Skrutskie M. F., et al., 2006, Astron. J., 131, 1163

Slumstrup D., Grundahl F., Silva Aguirre V., Brogaard K., 2019, A\&A, 622, A111

Stan Development Team 2018

Stassun K. G., Torres G., 2018, Astrophys. J., 862, 61

Stello D., et al., 2013, Prog. Phys. Sun Stars New Era HelioAsteroseismol., 479, 167

Stello D., et al., 2015, Astrophys. J., 809, L3

Travis E O., 2006, USA: Trelgol Publishing

Udalski A., 2000, Astrophys. J., 531, L25

Viani L. S., Basu S., Chaplin W. J., Davies G. R., Elsworth Y., 2017, The Astrophysical Journal, 843, 11

Vrard M., Mosser B., Samadi R., 2016, Astron. Astrophys., 588, A 87

Waskom M., et al., 2017, doi:10.5281/zenodo.883859

White T. R., Bedding T. R., Stello D., Christensen-Dalsgaard J., Huber D., Kjeldsen H., 2011, Astrophys. J., 743, 161

White T. R., et al., 2013, Mon. Not. R. Astron. Soc., 433, 1262

Yu J., Huber D., Bedding T. R., Stello D., Hon M., Murphy S. J., Khanna S., 2018, Astrophys. J. Suppl. Ser., 236, 42

Zinn J. C., Huber D., Pinsonneault M. H., Stello D., 2017, Astrophys. J., 844, 166

Zinn J. C., Pinsonneault M. H., Huber D., Stello D., 2018, ArXiv E-Prints, p. arXiv:1805.02650

This paper has been typeset from a TEX/LATEX file prepared by the author. 